\title{
A Modular Workflow for Model Building, Analysis, and Parameter Estimation in Systems Biology and Neuroscience
}

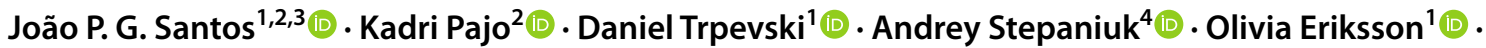 \\ Anu G. Nair ${ }^{2,5}$ (D) Daniel Keller ${ }^{4}$ (D) . Jeanette Hellgren Kotaleski ${ }^{1,2}$ (D) Andrei Kramer ${ }^{1}$ (D)
}

Accepted: 3 September 2021

(C) The Author(s) 2021

\begin{abstract}
Neuroscience incorporates knowledge from a range of scales, from single molecules to brain wide neural networks. Modeling is a valuable tool in understanding processes at a single scale or the interactions between two adjacent scales and researchers use a variety of different software tools in the model building and analysis process. Here we focus on the scale of biochemical pathways, which is one of the main objects of study in systems biology. While systems biology is among the more standardized fields, conversion between different model formats and interoperability between various tools is still somewhat problematic. To offer our take on tackling these shortcomings and by keeping in mind the FAIR (findability, accessibility, interoperability, reusability) data principles, we have developed a workflow for building and analyzing biochemical pathway models, using pre-existing tools that could be utilized for the storage and refinement of models in all phases of development. We have chosen the SBtab format which allows the storage of biochemical models and associated data in a single file and provides a human readable set of syntax rules. Next, we implemented custom-made MATLAB ${ }^{\circledR}$ scripts to perform parameter estimation and global sensitivity analysis used in model refinement. Additionally, we have developed a web-based application for biochemical models that allows simulations with either a network free solver or stochastic solvers and incorporating geometry. Finally, we illustrate convertibility and use of a biochemical model in a biophysically detailed single neuron model by running multiscale simulations in NEURON. Using this workflow, we can simulate the same model in three different simulators, with a smooth conversion between the different model formats, enhancing the characterization of different aspects of the model.
\end{abstract}

Keywords Interoperability $\cdot$ Multiscale modeling $\cdot$ SBtab $\cdot$ Global sensitivity analysis $\cdot$ Parameter estimation $\cdot$ Systems biology

\section{Introduction}

Computational systems biology is a data-driven field concerned with building models of biological systems. Methods from systems biology have proven valuable in

João P. G. Santos and Kadri Pajo contributed equally to this work

Jeanette Hellgren Kotaleski

jeanette@kth.se

$\triangle$ Andrei Kramer

andreikr@kth.se

1 Science for Life Laboratory, School of Electrical Engineering and Computer Science, KTH Royal Institute of Technology, 10044 Stockholm, Sweden

2 Department of Neuroscience, Karolinska Institute, 17165 Stockholm, Sweden neuroscience, particularly when studying the composition of synapses, molecular mechanisms of plasticity, learning and various other neuronal processes (Bhalla \& Iyengar, 1999; Hellgren Kotaleski \& Blackwell, 2010; Li et al., 2012). A wide variety of different software and toolboxes, each with their own strengths and weaknesses, are available

3 Graduate Program in Areas of Basic and Applied Biology, Abel Salazar Institute of Biomedical Sciences, University of Porto, Rua Jorge de Viterbo Ferreira 228, 4050-313 Porto, Portugal

4 Blue Brain Project, École Polytechnique Fédérale de Lausanne (EPFL), Lausanne, Switzerland

5 Department of Molecular Life Sciences, University of Zurich, Winterthurerstrasse 190, 8057 Zurich, Switzerland 
within the field. This diversity, however, can obstruct model reuse as interoperability between the different software packages and the convertibility between various file types is only solved in part. Interoperability can either mean that the model built in one simulator can be run in another or that both simulators interoperate at run-time either at the same or different scales (Cannon et al., 2007). The former is addressed by standardizing model descriptions for which notable examples in systems biology include the standard machine-readable model formats, such as XML-based SBML (Systems Biology Markup Language; Hucka et al., 2003) and CellML (Hedley et al., 2001), and human readable format, such as SBtab (Lubitz et al., 2016). An analogous model description language for neurons and networks is the NeuroML (Neural Open Markup Language; Gleeson et al. 2010, 2012).

We start by providing examples of available systems biology tools, for model building, parameter estimation and model analysis. We then proceed to describe our approach in developing a modular workflow to address some of the interoperability issues and present simulation results of an example use case in various simulators and frameworks, with further examples provided in Supplementary Materials. Our workflow starts with a human-readable representation of the model that is easily accessible to everyone and proceeds through various conversions into different simulation environments: MATLAB ${ }^{\circledR}$, COPASI, NEURON, and STEPS (STochastic Engine for Pathway Simulation). Specifically, we will describe the conversion tools we created for this purpose.

\section{Examples of Software and Toolboxes used in Systems Biology}

No software package is perfectly suited for every task, some have programmable interfaces with scripting languages, like the MATLAB® SimBiology® toolbox, some focus on providing a fixed array of functions that can be run via graphical user interfaces, like COPASI, although it now offers a Python toolbox for scripting (Welsh et al., 2018). Most toolboxes and software packages offer a mixture of the two approaches: fine-grained programmable interface as well as fixed high-level operations. At the extremes of this spectrum are powerful but inflexible high-level software on one side, and complex, hard to learn but very flexible libraries or toolboxes with an API (application programming interface) on the other. Some examples of general modeling toolboxes in MATLAB ${ }^{\circledR}$ are the SBPOP/SBToolbox2 (Schmidt \& Jirstrand, 2006), and the PottersWheel Toolbox (Maiwald \& Timmer, 2008). For Bayesian parameter estimation, there are the MCMCSTAT toolbox (Haario et al., 2006) in MATLAB ${ }^{\circledR}$, as well as pyABC (Klinger et al., 2018) and
pyPESTO (Schälte et al., 2020) in Python, and the standalone Markov Chain Monte Carlo (MCMC) software GNU MCsim that allows to estimate the posterior distribution by sampling a high-dimensional probability distribution (Bois, 2009). For global sensitivity analysis, there is the Uncertainpy Python toolbox (Tennøe et al., 2018). For simulations in neuroscience, some notable examples include NEURON (Hines \& Carnevale, 1997) and STEPS (Hepburn et al., 2012). Both are used for simulations of neurons and can include reaction-diffusion systems and electrophysiology.

These software packages do not all use the same model definition formats. Most have some compatibility with SBML, others use their own formats (e.g. NEURON uses MOD files). In some cases, an SBML file exported from one of these packages cannot be imported into another package without errors; so manual intervention may be required. ${ }^{1}$ Given an SBML file, a common task is to translate the contents into code that can be used in model simulations, for ordinary differential equations this is the right-hand side vector field function. There are several tools that facilitate the conversion between formats, e.g. the SBFC (The Systems Biology Format converter; Rodriguez et al., 2016) as well as the more general VFGEN (A Vector Field File Generator; Weckesser, 2008).

All toolboxes and software packages have great strengths and short-comings, and each programming language has different sets of (freely) available libraries which makes the development (or use) of numerical methods more or less feasible than in another language. One such example is the $\mathrm{R}$ package VineCopula for parameter dependency modeling, which has recently been used for modeling probability densities (in parameter spaces) between two MCMC runs (Eriksson et al., 2019). It infers the probability density function from a large enough sample, another method to do this is kernel density estimation. ${ }^{2}$ The VineCopula package implements a much more advanced and robust method of density estimation based on vines and copulas and performs well in high dimensional cases. This package is not easily replaced in many other languages. The Julia language, on the other hand, has a far richer set of differential equation solvers than R or MAT$\mathrm{LAB} \circledast$ and comes with very efficient forward sensitivity analysis methods.

Each researcher must therefore make decisions that result in the best compromise for them. If a researcher is familiar with a given set of programming/scripting languages it is probably not reasonable to expect them to be able to

\footnotetext{
1 for example: at the time of writing the SimBiology® toolbox (in MATLAB $($ ) exports the time variable as a normal variable, without the required definition URL, it must be added manually.

2 Kernel density estimation does not work very well in high dimensional problems.
} 
Fig. 1 Simplified scheme of the workflow. Thick arrows indicate steps for which we have developed automated tools. Text in bold refers to the generic components of the workflow and text in grey refers to existing software and data formats used in the current version of the suggested workflow

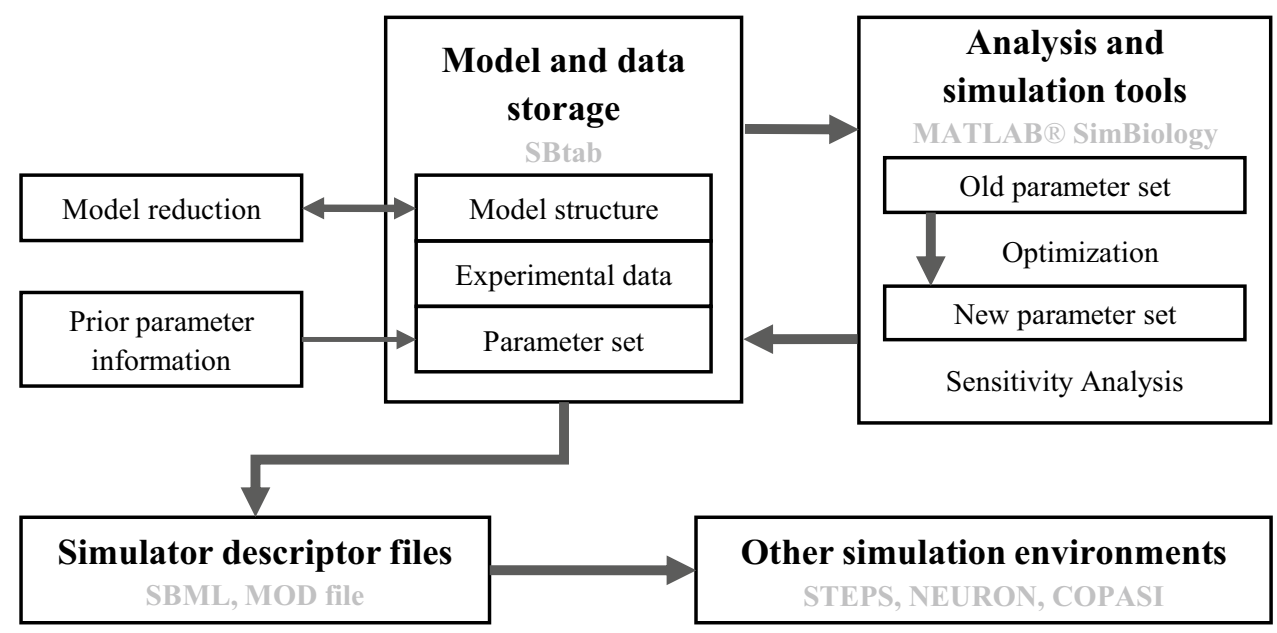

collaborate with other groups in languages they do not know. For this reason, it is our firm opinion that the conversion of models between different formats is a very important task, and it is equally important to use formats that people can pick-up easily. To make format conversion flexible intermediate files are a great benefit which leads to a modular approach with possible validation between modules.

We also have to consider the FAIR data principles-the findability, accessibility, interoperability and reusability of data and associated infrastructure (Wilkinson et al., 2016). Here, we would like to address the interoperability principle by having developed a workflow for building biochemical pathway models using existing tools and custom-made, short, freely available scripts for the storage and refinement of models in all phases of development, ensuring interchangeability with other formats and toolkits at every step in the pipeline using standardized intermediate files (Fig. 1 and Table 1).

Figure 1 illustrates the relationship between the tools we used in this workflow. We created a use case based on a previously developed model for others to reuse and modify, and we use it to demonstrate how the depicted parts operate. In addition, we made two other use cases available for testing other parts of the workflow. More extensive testing was done with a model of the mitogen-activated protein kinase (MAPK) cascade provided by the FindSim workflow (Viswan et al., 2018) and the results can be found in the Supplementary Materials.

\section{The Workflow}

While the standard model storage format in systems biology is SBML, it has some drawbacks: it does not lend itself to manual editing, the math in an SBML file is difficult to read and write manually, $\mathrm{xml}$ parsing is a difficult task that cannot be undertaken lightly by the novice programmer, species entries do not have a concentration unit attribute, time is handled very differently from any other model variable, etc. None of these issues are an error of course, but they are inconvenient for the inexperienced user. Therefore, this workflow is centered around building an easy-to-use infrastructure with models and data expressed in a spreadsheetbased storage format called SBtab (Lubitz et al., 2016). We chose SBtab as the primary modeling source file because it is human-readable and writable, it can contain both the model and the data, and because it is easy to write parsing scripts for it, such as a converter from SBtab to SBML using the libSBML ${ }^{3}$ interface in R. This ease of convertibility is used in the second focus of the workflow, convertibility between SBtab and other common formats and simulation software, since in systems biology and in any other computational sciences, the lack of compatibility between different tools and formats can often pose problems. A partially working conversion tool between SBtab and SBML had already been developed by the SBtab team. However, it can currently only read one table at a time and does not produce any functional SBML files with our model example. To combat these shortcomings, we wrote scripts to convert the SBtab into SBML, either using the R language or MATLAB $\AA$, and validating it successfully in COPASI, STEPS and NEURON.

The bulk of our workflow is available as MATLAB ${ }^{\circledR}$ code, particularly the parameter estimation tools and functions for global sensitivity analysis. Sensitivity analysis can be used to determine the importance of different parameters in regulating different outputs. Local sensitivity analysis is based on partial derivatives and investigates the behavior of the output when parameters are perturbed in close vicinity to a specific point in parameter space. Global sensitivity analysis, on the other hand, is based on statistical approaches and has a much broader range. Global sensitivity analysis

\footnotetext{
${ }_{3}$ A library providing an application programming interface for
} SBML. 
Table 1 An overview of available software packages and tools used by the Subcellular Workflow. Sometimes, manual intervention is needed in between workflow modules. This is especially true when an input format does not have a feature that an output format has. Addi- tionally, some software packages have so many functions that it could be easier to use them interactively (in these cases we also added a yes in the manual intervention column)

\begin{tabular}{|c|c|c|c|c|c|c|}
\hline & Tool & Interface Language & Purpose & Input Format(s) & Output Format(s) & Manual Intervention \\
\hline \multirow[t]{10}{*}{ Pre-existing tools } & MATLAB $® \operatorname{SimBiology}{ }^{\circledR}$ & MATLAB $®$ & $\begin{array}{l}\text { Simulations of biochemical } \\
\text { cascades }\end{array}$ & m, sbproj, SBML & m, sbproj, SBML & yes \\
\hline & $\begin{array}{l}\text { MATLAB® Optimization } \\
\text { Toolbox }{ }^{\mathrm{TM}}\end{array}$ & MATLAB® & Parameter estimation & none & none & no \\
\hline & COPASI & GUI & $\begin{array}{l}\text { Simulations of biochemical } \\
\text { cascades }\end{array}$ & $\begin{array}{l}\text { SBML, CPS, SED-ML, } \\
\text { COMBINE }\end{array}$ & $\begin{array}{l}\text { SBML, CPS, SED-ML, C, } \\
\text { COMBINE Archives, } \\
\text { XPPaut, Berkeley } \\
\text { Madonna }\end{array}$ & yes \\
\hline & NEURON & python & $\begin{array}{l}\text { Simulations of } \\
\text { electrophysiological neuron } \\
\text { models (with a possibility } \\
\text { to include biochemical } \\
\text { cascades) }\end{array}$ & $\bmod$ & not specific & yes \\
\hline & STEPS & python & $\begin{array}{l}\text { Stochastic simulations of } \\
\text { reaction-diffusion models on } \\
\text { tetrahedral meshes }\end{array}$ & python script & not specific & yes \\
\hline & NFsim & Shell & $\begin{array}{l}\text { Network-free and hybrid } \\
\text { simulations of rule-based } \\
\text { models }\end{array}$ & BNGL & not specific & yes \\
\hline & BioNetGen & GUI & $\begin{array}{l}\text { Environment for rule-based } \\
\text { model setup and simulation }\end{array}$ & BNGL & not specific & yes \\
\hline & VFGEN & Shell & $\begin{array}{l}\text { Converts ODE vector field files } \\
\text { (vf) to many other languages/ } \\
\text { formats }\end{array}$ & vf & .m,.R,.py,.c, many others & no \\
\hline & Conversion Tools & & & & & \\
\hline & $\underline{\text { SBML to SBtab }}^{\mathrm{a}}$ & web & $\begin{array}{l}\text { Conversion from SBML to } \\
\text { SBtab }\end{array}$ & SBML & SBtab & $\begin{array}{l}\text { Yes } \\
\text { (post-conversion) }\end{array}$ \\
\hline \multirow[t]{9}{*}{ Custom-developed tools } & Diagnostics tool $^{\mathrm{b}}$ & MATLAB® & $\begin{array}{l}\text { Runs the model, compares it } \\
\text { to the provided data and } \\
\text { calculates scores }\end{array}$ & SBtab as Excel & mat + figures & $\begin{array}{l}\text { not if SBtab is correctly } \\
\text { formatted }\end{array}$ \\
\hline & $\underline{\text { Parameter estimation }}^{\mathrm{b}}$ & MATLAB® & $\begin{array}{l}\text { Parameter estimation using } \\
\text { MATLAB® optimization } \\
\text { tools }\end{array}$ & SBtab as Excel & mat + figures & $\begin{array}{l}\text { not if SBtab is correctly } \\
\text { formatted }\end{array}$ \\
\hline & GSA analysis $^{b}$ & MATLAB® & $\begin{array}{l}\text { GSA analysis using Sobol- } \\
\text { Saltelli method implemented } \\
\text { in MATLAB® }\end{array}$ & SBtab as Excel & mat + figures & $\begin{array}{l}\text { not if SBtab is correctly } \\
\text { formatted }\end{array}$ \\
\hline & Simulation Setup Webapp $^{\mathrm{c}}$ & python & $\begin{array}{l}\text { Setup and run STEPS, } \\
\text { BioNetGen and NFsim } \\
\text { simulations }\end{array}$ & SBML, BNGL & BNGL, pySB & $\begin{array}{l}\text { yes, removal of functional } \\
\text { reaction rates and } \\
\text { applying stimuli }\end{array}$ \\
\hline & $\begin{array}{l}\text { get thermodynamic } \\
\text { constraints. }^{\mathrm{d}}\end{array}$ & GNU Octave & $\begin{array}{l}\text { Checks for thermodynamic } \\
\text { constraints between } \\
\text { parameters }\end{array}$ & $\mathrm{m}$ & Terminal text & yes \\
\hline & Conversion Tools & & & & & \\
\hline & $\underline{\mathrm{SBtab} \text { to SBML }+\mathrm{m}+\mathrm{tsv}^{\mathrm{b}}}$ & MATLAB® & $\begin{array}{l}\text { part of our MATLAB® } \\
\text { toolchain }\end{array}$ & SBtab as Excel & SBML, m, sbproj, tsv & no \\
\hline & $\underline{\text { sbtab to vfgen } .} R^{\mathrm{e}}$ & $\mathrm{R}$ & $\begin{array}{l}\text { Convert SBtab files into } \\
\text { VFGENs vf format, but also } \\
\text { produce SBML via libSBML }\end{array}$ & $\begin{array}{l}\text { SBtab as TSV } \\
\text { or ODS }\end{array}$ & $\begin{array}{l}\text { vf } \\
\text { SBML }\end{array}$ & $\begin{array}{l}\text { no } \\
\text { no }\end{array}$ \\
\hline & & & & & $\bmod$ & yes \\
\hline
\end{tabular}

${ }^{a}$ https://www.sbtab.net/sbtab/default/converter.html

${ }^{b}$ https://github.com/jpgsantos/Subcellular_workflow/blob/1.0/Matlab/Run_main.m

${ }^{c}$ https://subcellular.humanbrainproject.eu/model/simulations

${ }^{\mathrm{d}}$ https://github.com/jpgsantos/Subcellular_workflow/blob/1.0/Matlab/Code/Standalone/get_thermodynamic_constraints.m

${ }^{\mathrm{e}}$ https://github.com/a-kramer/SBtabVFGEN 
is more relevant for models that have a large uncertainty in their parameter estimates which is common for systems biology models where many of the parameters have not been precisely measured and the data are sparse.

A common approach within biochemical modeling is to use deterministic simulations and ordinary differential equations (ODE) that follow the law of mass action as it is computationally efficient and provides accurate (compared to averaged stochastic simulations) results for sufficiently large well-mixed biological systems. However, this approach has several restrictions in the case of neuronal biochemical cascades. First, such cascades are always subject to stochastic noise, which can be especially relevant in a compartment as small as a dendritic spine where the copy number of key molecules are small enough that the effect of randomness becomes significant (Bhalla, 2004). For precise simulation of stochasticity in reaction networks several stochastic solvers are available, e.g. Gillespie's Stochastic Simulation Algorithm (SSA) (Gillespie, 1976) and explicit and implicit tau-leaping algorithms (Gillespie, 2001). Second, the number of possible states of many biochemical cascades grow exponentially with the number of simulated molecule types, such that it becomes difficult to represent all these states in the model. In this case, for efficient simulation the reactions in the model could be represented and simulated in a network free form using rule-based modeling approaches (Chylek et al., 2015). Third, many biochemical networks are spatially distributed, this requires simulation of molecule diffusion (Hepburn et al., 2012). To tackle these problems, we developed the subcellular simulation setup application, a web-based software component for model development. It allows the extension and validation of deterministic chemical reaction network-based models by simulating them with stochastic solvers for reaction-diffusion systems (STEPS, Hepburn et al., 2012) and network free solvers (NFsim, Sneddon et al., 2011).

Regardless of the modeling approach (rule based or reaction network based ODE), the solvers yield a timecurve solution: $x(t ; \theta)$. This means that our workflow can only accept data that can be represented by one or several numerical solutions of this type. Time Series data and Dose Response curves can both be mapped to trajectory solutions. Dose Response curves are mapped point-by-point to solutions under varying input settings (doses). We have not used direct solvers to obtain steady states or other limit sets.

Although the workflow is in principle applicable to any biochemical pathway model our emphasis is on modeling biochemical signaling in neurons. Therefore, the last challenge we want to address is an important concept in the interoperability domain of computational neuroscience called multiscale modeling which concerns the integration of subcellular models into electrical models of single cells or in neuronal microcircuits. This can be achieved either by run-time interoperability between two simulators of different systems (Djurfeldt et al., 2010) or by expanding the capabilities of a single simulation platform as has been done with the NEURON software (McDougal et al., 2013). With this purpose in mind, we have written a conversion function from SBtab to the MOD format which is used by NEURON. As such, the inputs and the outputs of a biochemical cascade can be linked to any of the biophysiological measures of the electrical neuron model.

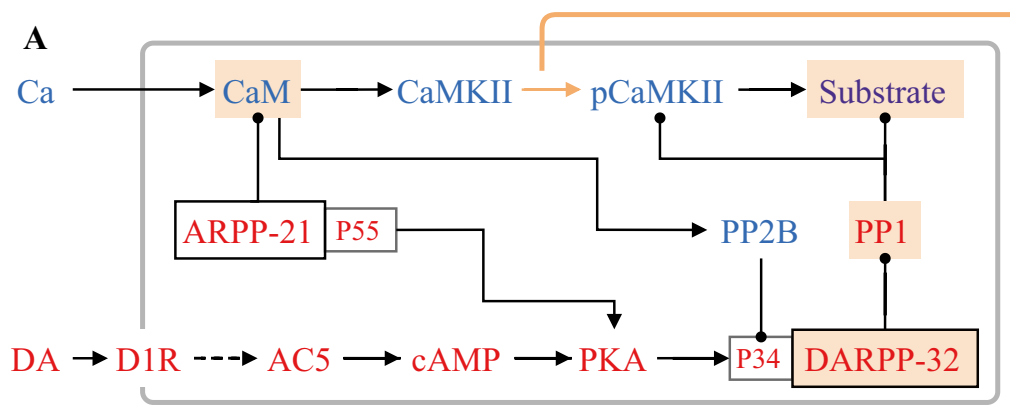

$\mathrm{C}$
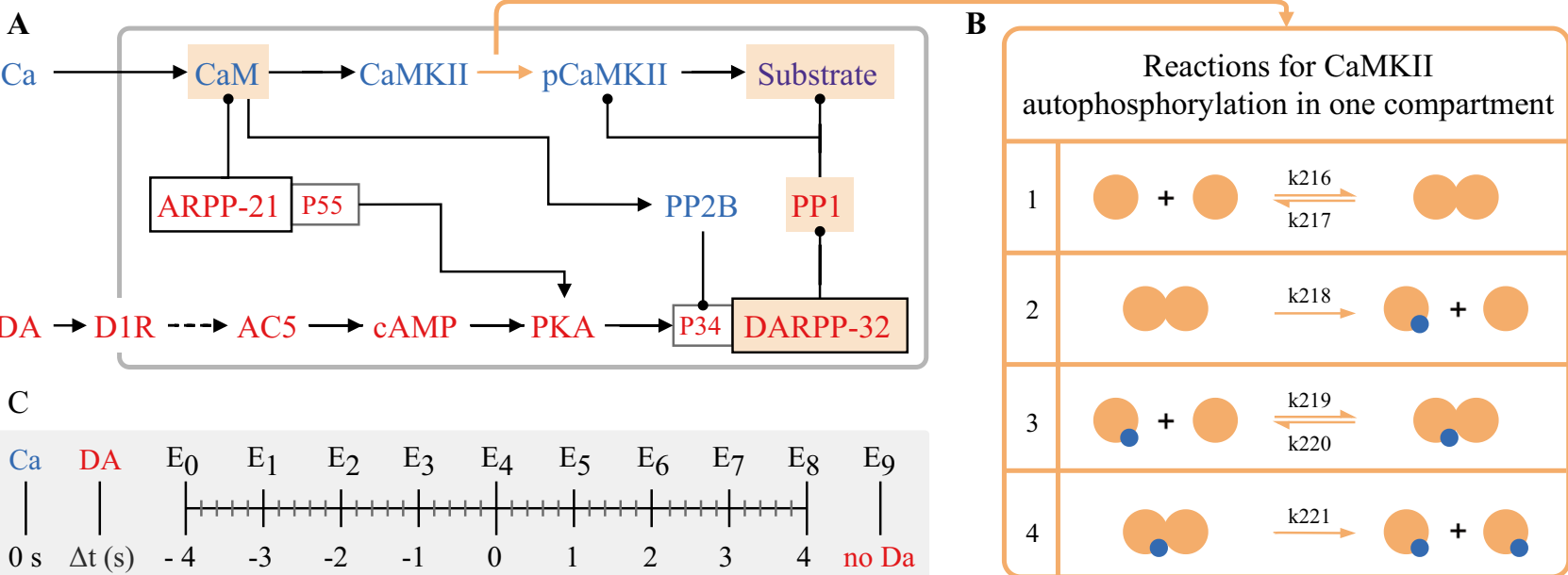

Fig. 2 A Simplified schematics of the use case model with relevant second messengers with calcium and dopamine as inputs and phosphorylation of a generic CaMKII substrate (purple; top right) as the output. Species of the calcium cascade are blue, and species of the dopamine cascade are red. Lines ending in arrows represent activation and lines ending in circles represent inhibition. Time courses of the species with a beige background are later used in parameter estimation. Readjusted from Nair et al. (2016). B Schematics of the bimolecular reactions used for CaMKII autophosphorylation with yellow circles depicting fully activated CaMKII bound to calmodulin and calcium, and blue circles depicting phosphate groups. Six newly introduced parameters are shown on the reaction arrows with their ID's in the updated model. C Timing (in seconds) of the dopamine input $(\Delta t=\{-4,-3,-2,-1,0,1,2,3,4\}$ corresponding to E0-E8) relative to the calcium input (zero), and a single experiment without a dopamine input (E9) 


\section{Use Case}

As a primary use case to illustrate the workflow we have chosen a previously developed pathway model of the emergence of eligibility trace observed in reinforcement learning in striatal direct pathway medium spiny neurons (MSN) that express the D1 receptor (Nair et al., 2016). Additional use cases are considered in the Supplementary Materials.

In this model, a synapse that receives excitatory input, which leads to an increase in calcium concentration, is potentiated only when the signal is followed by a reinforcing dopamine input. Figure 2A represents a simplified model scheme illustrating these two signaling cascades, one starts with calcium as the input and the other one with dopamine. In simulation experiments the inputs are represented as a calcium train and a dopamine transient (Fig. 3A). Calcium input refers to a burst of 10 spikes at $10 \mathrm{~Hz}$ reaching $5 \mu \mathrm{M}$. Dopamine input is represented by a single transient of $1.5 \mu \mathrm{M}$. The first cascade (species in blue) features the calcium-dependent activation of $\mathrm{Ca}^{2+}$ / calmodulin-dependent protein kinase II (CaMKII) and the subsequent phosphorylation of a generic CaMKII substrate which serves as a proxy for long term potentiation (LTP) and is the main output of the model. The second cascade (species in red) represents a G-protein dependent cascade following the dopamine input and resulting in the phosphorylation of the striatal dopamine- and cAMP-regulated phosphoprotein, $32 \mathrm{kDa}$ (DARPP-32) that turns into an inhibitor of protein phosphatase 1 (PP1) which can dephosphorylate both CaMKII and its substrate. The phosphorylation of the substrate is maximal when two constraints are met. First, the time window between the calcium and dopamine inputs has to be short, corresponding to the input-interval constraint which is mediated by DARPP-32 via PP1 inhibition. Second, intracellular calcium elevation has to be followed by the dopamine input, corresponding to the input-order constraint that is mediated by another phosphoprotein, the cyclic AMPregulated phosphoprotein, $21 \mathrm{kDa}$ (ARPP-21), thanks to its ability to sequester calcium/calmodulin if dopamine arrives first (Fig. 3D).
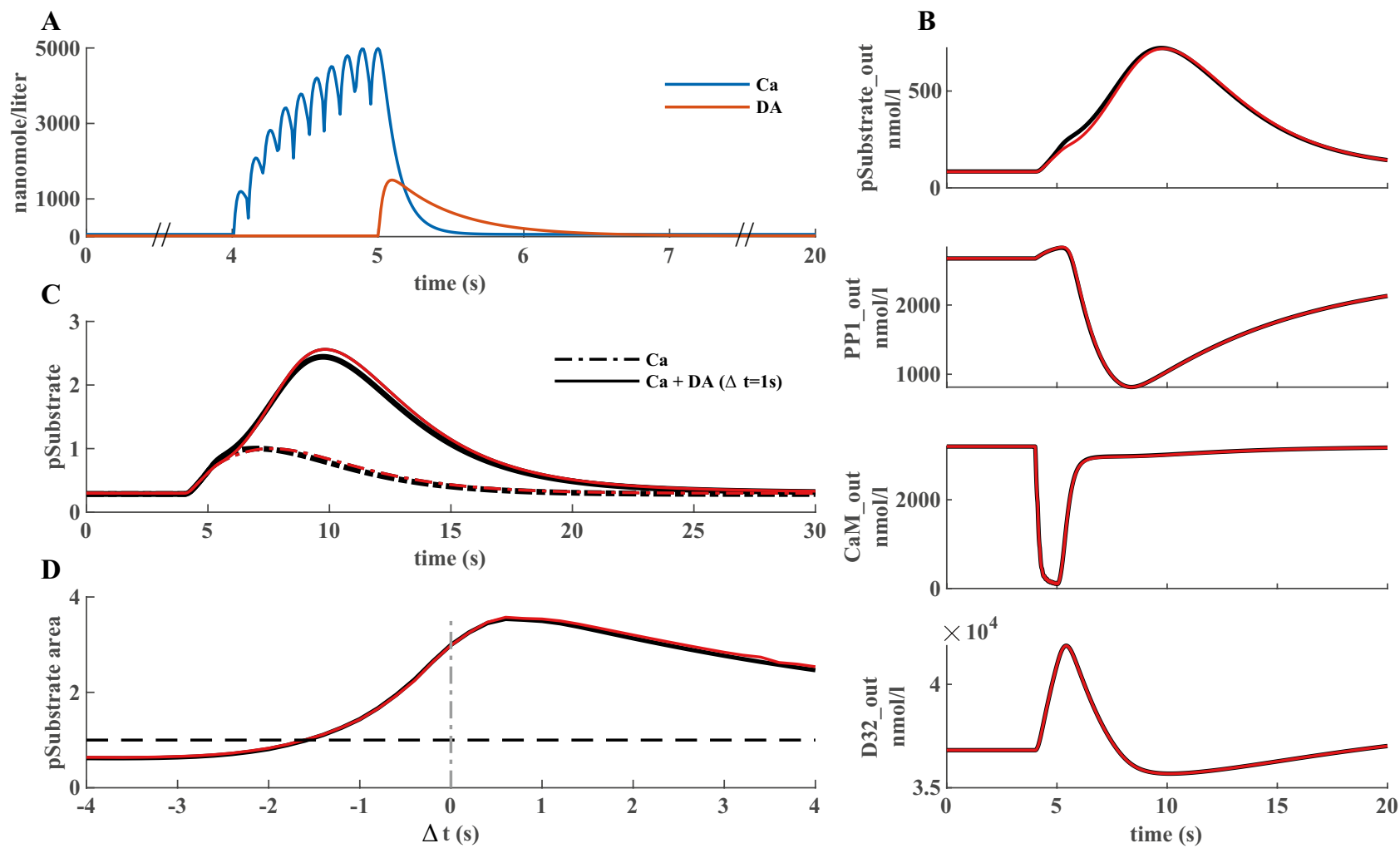

Fig. 3 A Illustration of the model inputs. Calcium burst (blue) at $4 \mathrm{~s}$ used in all simulations and a dopamine transient (orange) applied at different timings in eight experiments and one without it. B Four species used in parameter estimation corresponding to the input combination in A. Black traces represent the data produced by simulating the original model, red traces represent fits with the best new parameter sets in the updated model. C, D Comparison of model performance with substrate phosphorylation as the main model readout.
Here, $30 \mathrm{~s}$ simulations were used for comparison with the original model behavior. $\mathbf{C}$ Normalized time series of substrate phosphorylation, the main readout, with calcium as an only input or dopamine following it after 1s. D Normalized area under the curve of substrate phosphorylation with different calcium and dopamine input intervals. The simulations performed to obtain these graphs in MATLAB ${ }^{\circledR}$ took less than 5 min to compute (intel core i9-10980XE) 
In the originally published model, CaMKII is autophosphorylated in two compartments, both the cytosol and the post synaptic density (PSD), with a custom-written MAT$\mathrm{LAB} \AA$ rate function that was calculated based on the probability of two neighboring subunits being fully activated as described in Li et al. (2012). To make it possible to run the model in different software we replace the rate equation of autophosphorylation with a similar set of reactions in both compartments so that the model would only contain bimolecular reactions. The reactions represent a simplified version of the autophosphorylation reactions in Pepke et al. (2010), where in our case only the fully activated CaMKII can be phosphorylated. The same set of reactions is used in both compartments and the schematics is available in Fig. 2B along with the required six new parameters. We used our parameter estimation script to find parameter values and bounds that preserved the qualitative behavior of the model. In this primary use-case, we used simulated data (real data is used in the supplementary materials model) from the original model with different timings of the dopamine input relative to the calcium input (Fig. 2C) to obtain a comprehensive picture of its behavior which we want the updated model to reproduce.

\section{SBtab}

As described above we have chosen SBtab as the format at the root of our workflow for the storage of the model and associated data. In this section we illustrate how we use this format. More information, documentation and examples are available from the authors of SBtab. ${ }^{4}$ SBtab allows the storage of biochemical models and associated data in a single file and provides a set of syntax rules and conventions to structure data in a tabulated form making it easy to write, modify and share. To ensure interoperability, SBtab provides an online tool to convert the models into the SBML format. ${ }^{5}$ SBtab is suitable for storing data that comes in spreadsheet or table formats, e.g. concentration time series or dose response curves, but it is likely that any data format that can be reasonably stored as a table will work well in SBtab. The SBtab file is intended to be updated manually during the process of model building. Additional instructions on how to make SBtab files work well within our toolchain can be found in the Subcellular Workflow documentation. ${ }^{6}$ Some of the columns and sheets that we use should be considered as extensions to the format and are discussed in the documentation. SBtab is easy to parse so adjustments to parsers can be made quickly.

\footnotetext{
${ }^{4}$ https://sbtab.net/

5 https://humanbrainproject.github.io/hbp-sp6-guidebook/online_ usecases/subcellular_level/subcellular_app/subcellular_app.html

${ }^{6}$ https://subcellular-workflow.readthedocs.io/en/master/SBtab.html
}

The SBtab file should include separate sheets for compartments, compounds, reactions, assignment expressions, parameters, inputs, outputs, and experiments (as well as data tables). We illustrate the functionalities of SBtab here with the use case. The use case model has 99 compounds, 138 reactions and 227 parameters. An example of the SBtab reaction table can be found in Table 2 .

One of our goals with this study was to reproduce the original model behavior after replacing a single module inside the model to convert it to bimolecular reactions only. The data we used therefore represents the simulated time series (20 s) of the concentrations of four selected species in response to different input combinations using the original model. Each individual data sheet (named E0-E9, Fig. 2C) in SBtab represents the outputs of one experiment. A separate sheet called Experiments allows to define the input parameters differently for each experimental setup. By setting the initial concentrations of the unused species to zero the data could be mapped to a specific sub-module of the model (the remaining species). In this case the initial conditions are the same for all experiments. The Experiments table can also support annotations relevant to each dataset. We used nine different timings (corresponding to E0-E8) between the calcium and the dopamine signal starting with a dopamine signal preceding calcium by four seconds and finishing with dopamine following calcium after four seconds as this corresponds to the time frame originally used in model development $(\Delta t=\{-4,-3,-2,-1,0,1,2,3,4\})$. Additionally, we used simulations with calcium as the only input (E9) (Fig. 2). The time series of the input species are in a separate sheet following each experiment sheet. An example of how experimental data is stored can be found in Table 3 .

\section{Model Pre-Processing Tools}

Model building entails frequent changes to the model structure by adding new species, reactions, and parameters. This can result in the emergence or disappearance of Wegscheider cyclicity conditions that refer to the relationships between reaction rate coefficients arising from conditions of thermodynamic equilibrium (Wegscheider, 1901; Vlad \& Ross, 1994). Identified thermodynamic constraints show parameter dependencies that follow from physical laws and can reduce the number of independent parameters. These conditions are frequently difficult to determine by human inspection, especially for large systems. Similarly, identifying conserved moieties, like conserved total concentration of a protein, allows the reduction of the ODE model size, which leads to increased performance. In order to address these model pre-processing needs our toolkit includes scripts in MATLAB ${ }^{\circledR} /$ GNU Octave that use the stoichiometric matrix of the reaction network as an input to determine the thermodynamic constraints as 


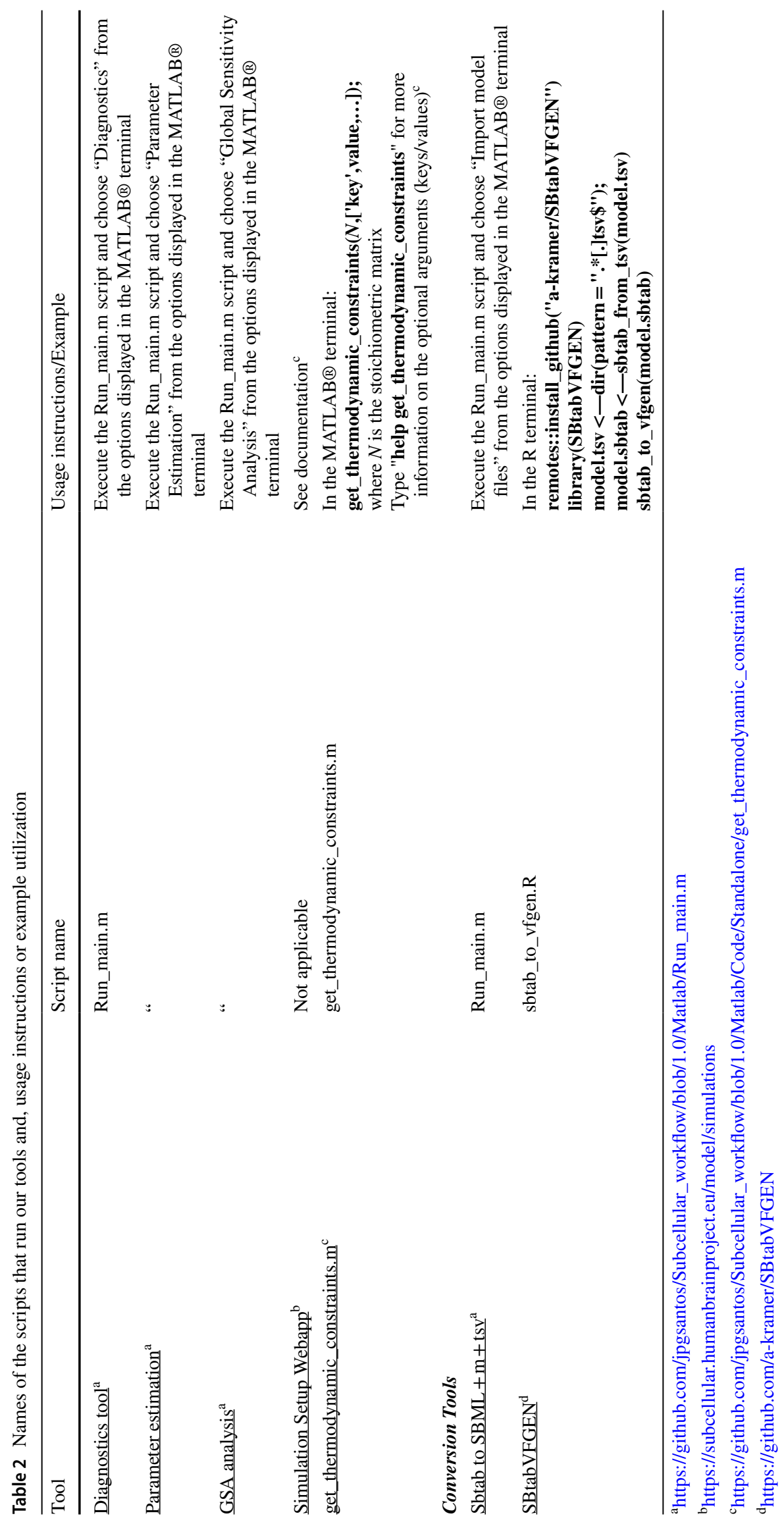



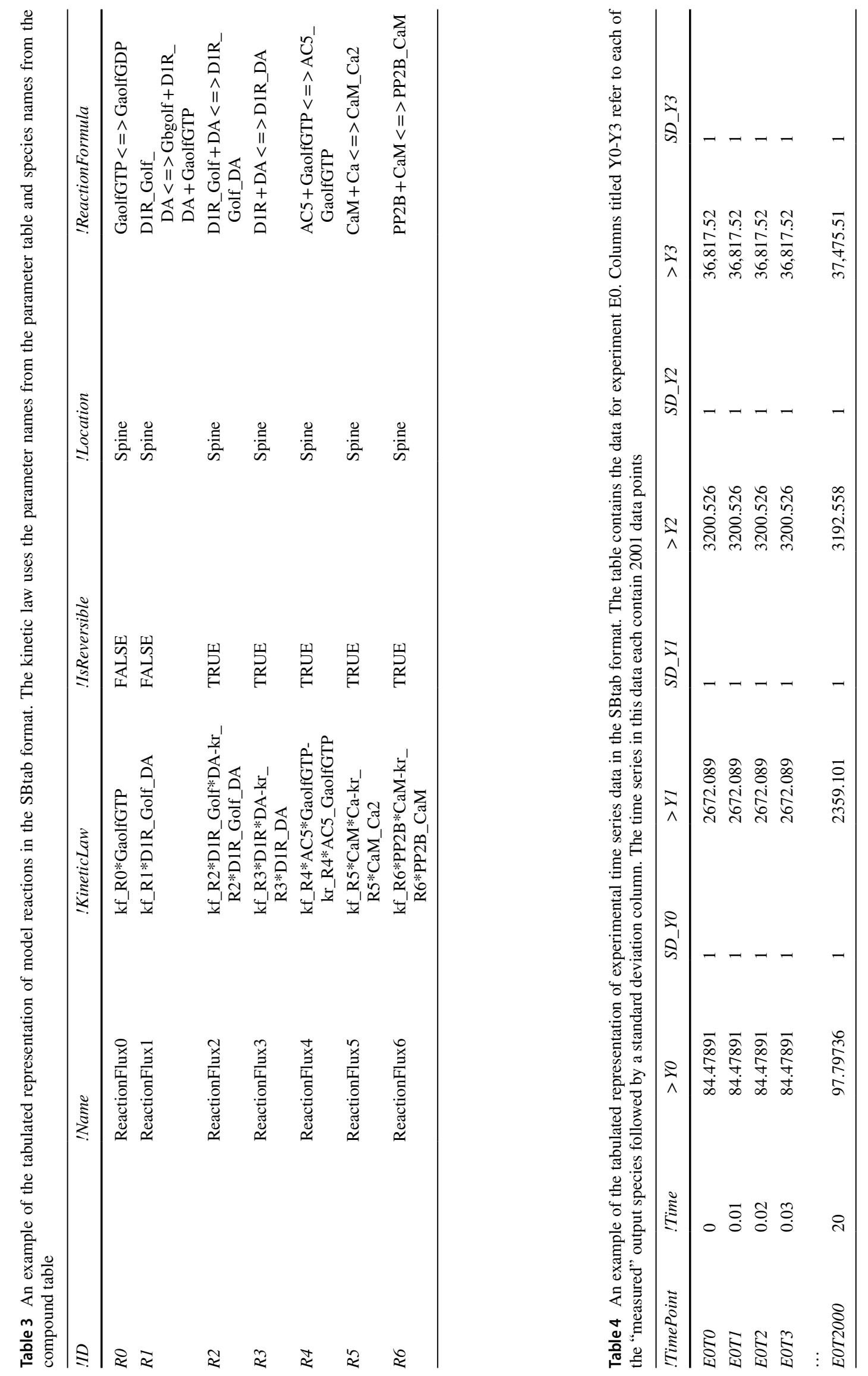
described in Vlad and Ross (1994), and conservation laws (Tables 1 and 4). These diagnostic tools output any identified constraints that, if needed, are to be implemented manually before the parameter estimation step. It should be noted that such constraints need to be re-examined after each addition of new reactions as the structure of the model might change and make previously true constraints invalid. This is true for all major changes to the model.

\section{MATLAB $^{\circledast}$ Tools}

The bulk of our workflow is developed in MATLAB $®$ as it provides an easy-to-use biochemical modeling application with a graphical user interface called SimBiology ${ }^{\circledR}$ along with a wide range of toolboxes for mathematical analysis. The workflow is divided into import and analysis scripts. We have written software for three types of analysis: diagnostics tools which are used to run the model and visualize how the model fits the data, parameter estimation, and global sensitivity analysis. To ensure an easy and user-friendly usage, all operations are controlled by a single settings file where all specification options needing user input are represented as modifiable variables. An example settings file of the use case model along with instructive comments can be found in the model GitHub repository. ${ }^{7}$ Only this settings file and the model in the SBtab format are needed as input from the user to run all our MATLAB ${ }^{\circledR}$ scripts. After running the analysis, the inputs and the data points along with the simulated model fits are plotted and the results are stored inside the model folder. This process is entirely automatic, but the user can always explore and retrieve more data from the created files. For example, after running the parameter estimation analysis, a plot is generated with the original parameter set, the prior bounds, and optimized parameters, but the procedure for retrieving the optimized parameters and using them to create a new SBtab or a new settings file for subsequent runs is not yet automated. For additional explanations of these functionalities please see our GitHub repository documentation. ${ }^{8}$

\section{Import from SBtab to MATLAB ${ }^{\circledR}$}

The import tools we have developed generate all the model and data files that are needed to run any of the analysis options in MATLAB $®$ that can be found in Table 1. These files are saved in subfolders of the main model that are created at run time. The files include a version of the model without any inputs in the MATLAB ${ }^{\circledR}$ (.mat) and SimBiology ${ }^{\circledR}$ (.sbproj) format, as well as several versions of the

\footnotetext{
7 https://github.com/jpgsantos/Model_Nair_2016/tree/1.0/Matlab/ Settings

8 https://subcellular-workflow.readthedocs.io/
}

model corresponding to each experiment specified in the SBtab. The latter includes three versions of the model that are used for different purposes: equilibration, default and detailed. Equilibration does not have any inputs and is used to equilibrate the species, whereas the default and detailed versions are used for simulation of experiments. They have all the relevant inputs and outputs that are going to be measured when simulating an experiment, and only differ in the step size of the simulations (both of which can be chosen in the settings file with detailed usually being a smaller step size). Additionally, while not needed for the rest of our MATLAB $®$ workflow, these import scripts also generate .tsv files corresponding to the individual SBtab sheets (useful for tracking changes in GitHub), and an SBML file using MAT$\mathrm{LAB}{ }^{\circledR}$ built-in functions (level 2 version 4 encoding as of MATLAB ${ }^{\circledR}$ 2021a). The latter can be used by any simulator that can import SBML files but requires further processing for which an R script can be found in a separate repository. ${ }^{9}$ Note that we have another converter from SBtab into SBML that runs in R instead of MATLAB ${ }^{\circ}$. We also generate other helper files that assist in the correct simulation of the MATLAB ${ }^{\circledR}$ model. A description of these and a more detailed explanation of the organization and of the created files and folders can be found in our documentation. ${ }^{10}$

\section{Parameter Estimation}

MATLAB ${ }^{\circledR}$ offers a wide range of tools for function optimization. We have developed scripts that transform our parameter estimation problem into an objective function that can be optimized by various MATLAB $®$ built-in optimizer functions. At the time of writing, these include "fmincon", simulated annealing ("simulannealbnd"), pattern search ("patternsearch"), genetic algorithm ("ga"), particle swarm ("particleswarm"), and surrogate optimization algorithms ("surrogateopt"), for which MATLAB ${ }^{\circledR}$ provides thorough documentation. The code is built with flexibility in mind, so introduction of other MATLAB ${ }^{\circledR}$ built-in or custom optimization algorithms should be straightforward. The optimizers used are the ones chosen in the settings file. Our code supports the use and comparison of multiple optimizers at the same time, and multiple uses of the same optimization algorithm are also supported. This is particularly useful for using optimizers that are inherently single-threaded, e.g. the simulated annealing algorithm, since multiple simulated annealing optimizations can be performed in multiple computing cores. After performing the optimization, a file containing the results is produced from which the optimized outputs can be retrieved and used to manually update the

\footnotetext{
9 https://github.com/a-kramer/simbiology-sbml-fix

${ }^{10}$ https://subcellular-workflow.readthedocs.io/en/master/Files.html
} 
SBtab or SimBiology ${ }^{\circledR}$ model. One of the equations used to calculate the score for how well the model outputs fit the experimental data can be found below (Eq. 1). We have incorporated a few other ways of calculating the score, and custom scoring methods could also be added depending on the need (see documentation).

$F(\theta ; Y, \tau)=\sum_{k=1}^{l} \sum_{j=1}^{m} \frac{1}{n} \sum_{i=1}^{n}\left(\frac{Y_{i j k}-y_{i j k}(\theta)}{\tau_{i j k}}\right)^{2}$

Here, $Y$ represents the data that is going to be used to constrain the model, sourced either from experiments or previous models, and $y$ represents the outputs of the model mapped to the data resulting of the simulation of the model under parameterization $\theta$. The allowed mismatch $\tau$ between the two simulation results is analogous to the standard deviation of a Gaussian noise model in data fitting. The resulting $F$ is the objective function for optimization. The error is summed over $n$, the number of points in each experimental output, $m$, the number of experimental outputs in an experiment (which is four in our use case, see Fig. 3B), and $l$, the number of experiments (E0-E9 in our use case) (see Fig. 2C).

Parameter estimation is generally based on experimental data. In this use case, however, we used simulated data of the concentrations of several species using the original version of the model in SimBiology® (the additional use cases provided in the Supplementary Materials use actual experimental data). After modifying the model, we minimized the difference between the old behavior and the updated model's response through optimization. The simulation results from the old model can be considered as analogous to experimental data in a normal parameter estimation setting. Here, we merely aim to make an updated model agree with its earlier iteration, which itself was adjusted based on experimental data. When changing a module in a model it is crucial to protect the unchanged parts, which is why we performed parameter estimation using the key species that intersect the calcium and dopamine cascades, namely PP1, calmodulin and DARPP-32 (Fig. 2 and Fig. 3B). In this use case, the Particle Swarm Algorithm was chosen to perform the optimization, but all algorithms were capable of reasonable optimizations. The parameters obtained were then used to generate all the figures where optimized parameters are referred to. The choice of total amount of reactions, used to replace the original function that represented the CaMKII phosphorylation, were constrained by the optimization. We considered the outputs that we were measuring (Fig. 3B) and added reactions until the addition of more did not meaningfully improve the fits.

\section{Validation in MATLAB ${ }^{\circledR}$}

We developed diagnostics scripts that can be used to reproduce the various experiments defined in SBtab. These scripts generate plots of the experimental inputs to the model (adapted for Fig. 3A), the provided data and the outputs measured from model simulation (adapted for Fig. 3B) given some choice of parameters, and plots of the scores calculated for the differences between the various experimental outputs and simulated model outputs. We used these tools to confirm that our parameter estimation resulted in a good fit for most of the species and the updated model was able to closely reproduce the results seen with the original model (Fig. 3C). In our repository we provide the updated model in SBtab (.xlxs and.tsv), SBML (.xml) and MATLAB ${ }^{\circledR}$ SimBiology® (.sbproj and.mat). In addition to the generalpurpose tools, we also wrote a use case-specific script, which uses data from the original model and reproduces the timedependency of the substrate phosphorylation given different delays of the start of calcium and dopamine stimuli, using the optimized model (Fig. 3C, D).

\section{Global Sensitivity Analysis}

In many cases parameter estimation of biochemical pathway models does not result in one unique value for a parameter. Structural and practical unidentifiability (Raue et al., 2009) results in a large set of parameter values that all correspond to solutions with a good fit to the data, i.e., there is a large uncertainty in the parameter estimates (Eriksson et al., 2019). When this is the case, local sensitivity analysis is not so informative, since this can be different depending on which point in parameter space it is performed at. A global sensitivity analysis (GSA), on the other hand, covers a larger range of the parameter space. Several methods for GSA exist ( $\mathrm{Zi}$, 2011) but we have focused on a method by Sobol and Saltelli (Saltelli, 2002, 2004; Sobol, 2001) as implemented by Halnes et al. (2009) which is based on the decomposition of variances (Saltelli, 2004). Single parameters or subsets of parameters that have a large effect on the variance of the output get a high sensitivity score in this method. Intuitively, this method can be understood as varying all parameters but one (or a small subset) at the same time within a multivariate distribution to determine what effect this has on the output variance. If there is a large reduction in the variance, the parameter that was kept fixed is important for this output (Saltelli, 2004).

Let the vector $\Theta$ denote the parameters of the model, and $y=f(\Theta)$ be a scalar output from the model. In the sensitivity analysis $\Theta$ are stochastic variables, sampled from a multivariate distribution, whose variation gives a corresponding uncertainty of the output, quantified by the variance $V(Y)$. Note that in the setting and interpretations described here, the different $\Theta_{i}$ are assumed to be independent from each other (for cases with dependent $\Theta_{i}$ see e.g. (Saltelli, 2004) and (Eriksson et al., 2019)). We consider two types of sensitivity indices: the first order effects $S_{i}$ and the total order 
$\mathbf{A}$

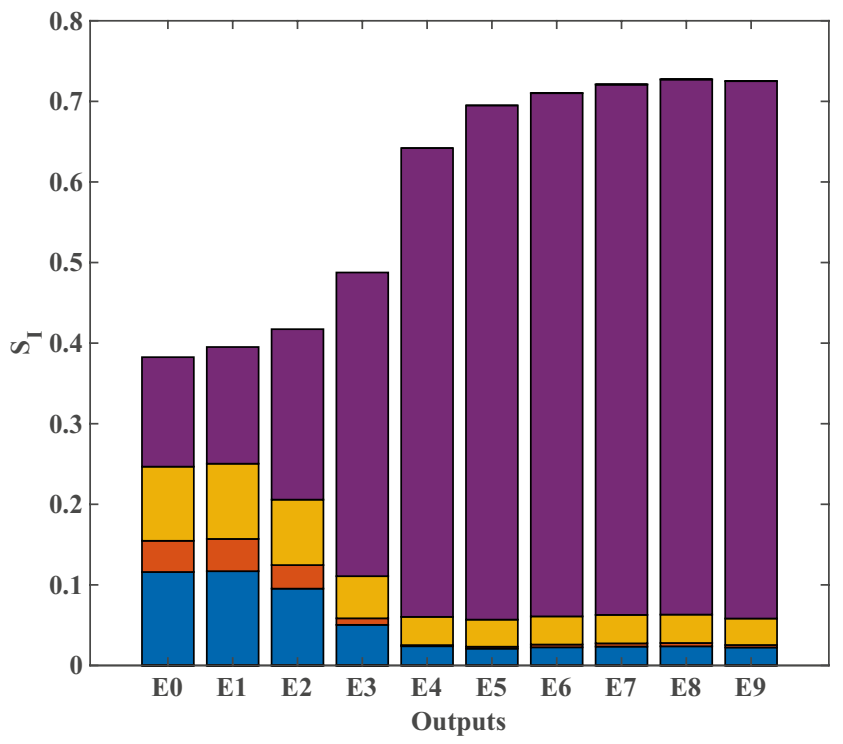

B

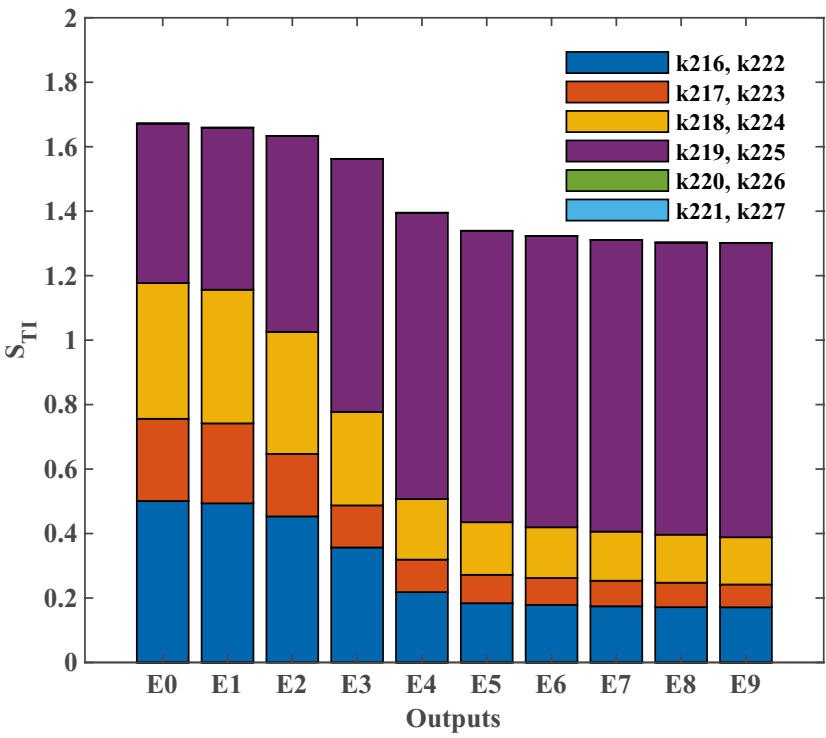

Fig. 4 Stacked bar graphs of the sensitivity indices. The first order, $\mathrm{S}_{\mathrm{i}}$, and total order, $\mathrm{S}_{\mathrm{Ti}}$, sensitivities indices of the six new parameters (k216-k221 and k222-k227; see Fig. 2) for all ten experiments (E0E9) are shown (panel A and B, respectively). The sensitivities indices are defined in the main text and were calculated based on the scores used in the optimization for each experiment respectively. The parameters, $\Theta$, were sampled independently from a multivariate lognormal distribution with $\log 10(\Theta) \sim \mathrm{N}(\mu, \sigma)$, using $\mu=\log 10\left(\Theta^{*}\right)$ and $\sigma=0.1$,

effects $S_{T i}$. The first order effects describe how the uncertainty in the output depends on the parameter $\Theta_{i}$ alone, i.e., how much of the variance of the output can be explained by the parameter $\Theta_{i}$ by itself. As an example, $S_{i}=0.1$ means that $10 \%$ of the output variance can be explained by $\Theta_{i}$ alone. The total order effects give an indication on the interactive effect the parameter $\Theta_{i}$ has with the rest of the parameters on the output. Parameters are said to interact when their effect on the output cannot be expressed as a sum of their single effects on the output.

The first order sensitivity index of the parameter $\Theta_{i}$ is defined as ${ }^{11}$

$S_{i}=\frac{V_{\Theta_{i}}\left[E_{\Theta_{-i}}\left[Y \mid \Theta_{i}\right]\right]}{V[Y]}, \quad \sum_{i} S_{i} \leq 1$,

where $\Theta_{-i}$ corresponds to all elements of $\Theta$ except $\Theta_{i}$ The total order sensitivity index of the parameter $\Theta_{i}$ is defined as

$S_{T i}=1-\frac{V_{\Theta_{-i}}\left[E_{\Theta_{i}}\left[Y \mid \Theta_{-i}\right]\right]}{V[Y]}, \quad \sum_{i} S_{T i} \geq 1$,

If there is a large difference between $\mathrm{S}_{i}$ and $\mathrm{S}_{\mathrm{Ti}}$, this is an indication that this parameter takes part in interactions. For a detailed description see chapter 5 of Saltelli (2004).

11 Where \$ $\mathrm{V} \$ \$$ is the variance operator and \$\$E\$ (conditional) expected value. where $\Theta^{*}$ correspond to the optimal values received from the optimization. A sample size of $\mathrm{N}=10,000$ was used (corresponding to 80,000 reshuffled samples used in the calculations (Saltelli, 2004)). The analysis took $3 \mathrm{~h} 30 \mathrm{~min}$ using an intel core i9-10980XE. The sample size was chosen big enough to make the differences in the sensitivity scores stemming from different seeds 18 small for the purposes of our conclusions

The optimization described earlier takes place on log transformed space $\left(\log _{10}(\Theta)\right)$. For the sensitivity analysis we perform the sampling on a lognormal distribution, meaning that $\log _{10}(\Theta) \sim N(\mu, \sigma)$. Below we use $\mu=\log _{10}\left(\Theta^{*}\right)$ and $\sigma=0.1$, where $\Theta^{*}$ correspond to the optimal values received from the optimization. ${ }^{12}$ We illustrate this method using only the six parameters corresponding to the model module that has been replaced (Fig. 2) and the results can be seen in Fig. 4 Only four of the parameters (k216-k219/k222-k225; Fig. 4) seem to be important for the output within the investigated parameter region, with the parameter k219/k225 dominating in experiments E3 to E9. There also seem to be some interactive effects between the parameters since $\mathrm{S}_{\mathrm{Ti}}$ is larger than $\mathrm{S}_{\mathrm{i}}$, especially for the first four experiments (Fig. 4A, B).

\section{Compatibility and Validation with Other Simulation Environments}

\section{Conversion to SBML and Simulations in COPASI}

COPASI is one of the more commonly used modeling environments in systems biology and it can read SBML files

$\overline{12}$ Other distributions can be used as well. 


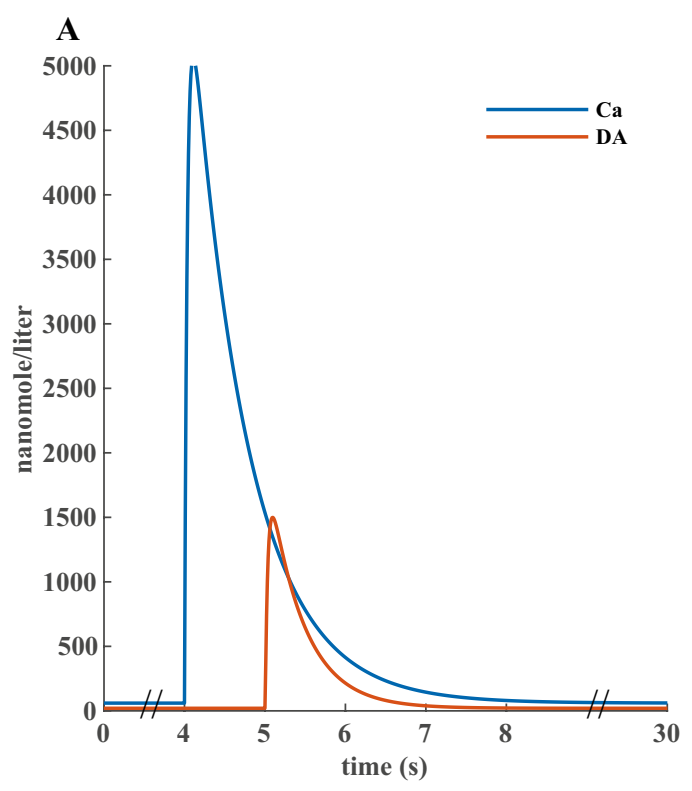

Fig. 5 Simulations in identical conditions in both MATLAB ${ }^{\circledR}$ SimBiology ${ }^{\circledR}$ and COPASI yielded almost identical results. A Inputs used in both simulators. The calcium input is kept constant at $4 \mathrm{~s}$ for all simulations and dopamine input time is varied from time 0 to $8 \mathrm{~s}$ at every one second. The difference from the previous simulations is in the calcium input which, for the sake of simplicity, is represented
B
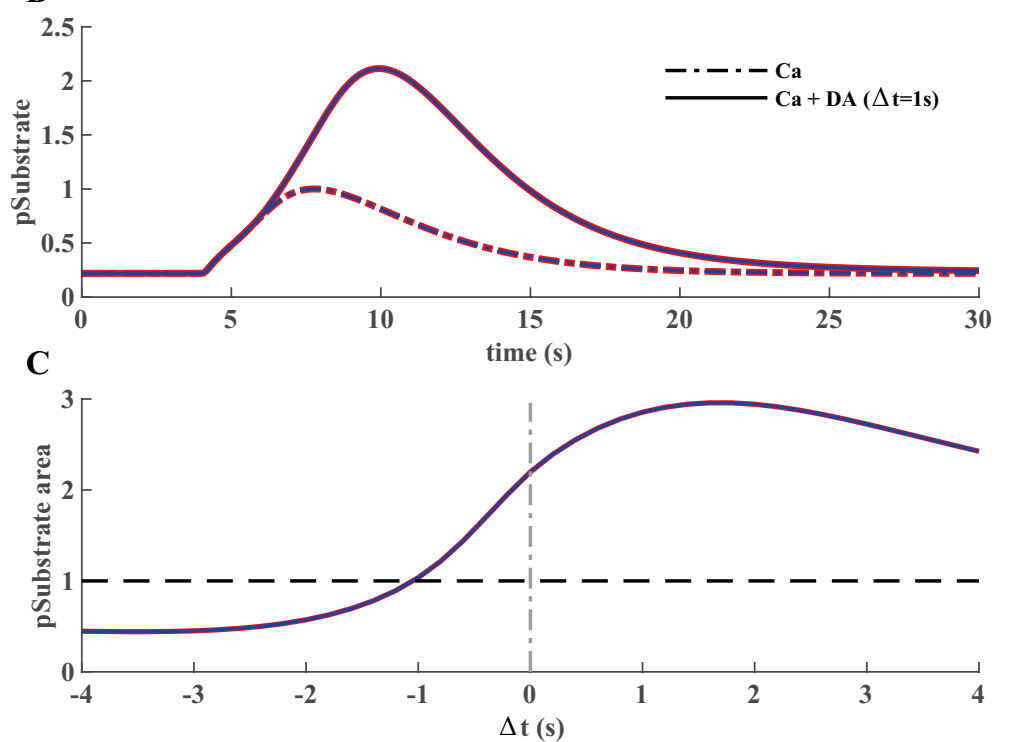

(Hoops et al., 2006). Our first validation step is to use the SBML model retrieved from the SBtab model in COPASI. The online conversion tool from SBtab to SBML did not work for our specific use case; we wrote a new conversion function that can be found in a separate GitHub repository. ${ }^{13}$ It interprets the biological model and converts it into plain ODEs in VFGEN's custom format (.vf), the VFGEN file can then be used to create output in various languages ${ }^{14}$ (Weckesser, 2008). The conversion script (written in R) converts the SBtab saved as a series of .tsv files or one .ods file into a VFGEN vector field file and as by-products also the SBML and a MOD file (see chapter Conversion to a MOD file and simulations in NEURON). To create an SBML model, libsbml must be installed with $\mathrm{R}$ bindings. The SBML file can be imported directly into COPASI.

Another way to convert the model into SBML is through a single MATLAB® SimBiology® function. The models, however, are created with long ID's that carry no biological information (the IDs are similar to hexadecimal hashes) for all model components, the units are not properly recognized,

\footnotetext{
$\overline{13}$ Conversion function available in https://github.com/a-kramer/ SBtabVFGEN and instructions in https://github.com/a-kramer/ SBtabVFGEN/blob/master/README.md

14 Including, but not limited to: Python (NumPy), C (GNU Scientific Library (GSL), CVODE), GNU Octave (LSODE), R (deSolve), and core MATLAB ${ }^{\circledR}$ (e.g. for ode15s).
}

by a double exponential spike. $\mathbf{B}$ and $\mathbf{C}$ show substrate phosphorylation curves analogous to Fig. 3, the red line represents results obtained in MATLAB $®$ and blue line results from simulations in COPASI. A single $30 \mathrm{~s}$ simulation took around $10 \mathrm{~s}$ of compute time within a $1 \mathrm{fl}$ spine volume (Intel ${ }^{\circledR}$ Core $\left.^{\mathrm{TM}} \mathrm{i} 7-8750 \mathrm{H}\right)$

and some units may just be incorrectly defined in the output. $\mathrm{We}$, therefore, created a script in $\mathrm{R}$ that asks for default units for the model and replaces the ones in SimBiology's SBML file. It fixes most issues (units, IDs, and the time variable in assignments) allowing the model to be properly imported in COPASI. ${ }^{15}$ To illustrate that the SBML-converted model imported into COPASI produces the same results as in SimBiology®, we used a simplified calcium input corresponding to one double exponential spike analogous to the dopamine transient and simulated the model with deterministic solvers in both COPASI (LSODA solver) and MATLAB $®$ SimBiology ${ }^{\circledR}$ under similar conditions. Both simulation environments produced almost overlapping results (Fig. 5B, C) validating the converted model in the SBML format.

\section{Simulations in STEPS}

The web-based subcellular simulation setup application ${ }^{16}$ allows importing, combining and simulating models expressed in the BioNetGen language (BNGL; Harris et al.,

\footnotetext{
${ }^{15}$ It should be noted that the units may not show up correctly in COPASI (depending on the version) even if they are correct in the SBML file itself.

16 The online application for subcellular simulations can be found in https://subcellular.humanbrainproject.eu/model/simulations with the documentation in https://humanbrainproject.github.io/hbp-sp6-guidebook/ online_usecases/subcellular_level/subcellular_app/subcellular_app.html
} 


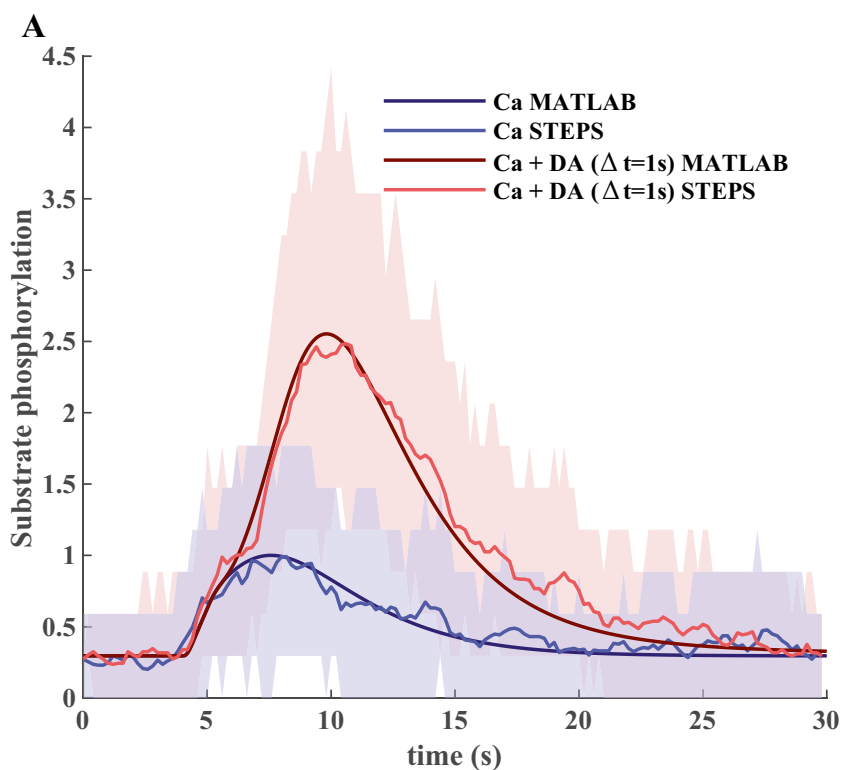

Fig. 6 Validation of the model by stochastic STEPS simulation of substrate phosphorylation in a typical D1 MSN spine. A Normalized time course of substrate phosphorylation in the updated model run in MATLAB ${ }^{\circledR}$ in comparison with averaged $(n=50)$ stochastic STEPS simulations (red-calcium and dopamine; blue-calcium only) for a typical size of D1 MSN synaptic spine $(\mathrm{V}=0.02 \mu \mathrm{m} 3)$. The same stimulation protocol as in Fig. 3 was used. Colored areas around averaged STEPS curves correspond to a range between 10 and $90 \%$ confidence intervals. One simulation required less than 1 min of compute time for $30 \mathrm{~s}$ of simulated reactions within a $0.02 \mu \mathrm{l}$ spine

2016). It supports the import of SBML (level 2 version 4) models and their transformation to rule-based BNGL form using Atomizer (Tapia \& Faeder, 2013). The BioNetGen file format was extended to provide diffusion parameters, links to tetrahedral meshes describing the geometry of model compartments, as well as the additional parameters for solvers and stimulation protocols required for spatially distributed models. The subcellular simulation setup application is integrated with the network free solver NFsim (Sneddon et al., 2011) and it supports simulations of spatially distributed systems using STEPS (Hepburn et al., 2012). STEPS provides spatial stochastic and deterministic solvers for simulations of reactions and diffusion on tetrahedral meshes. Furthermore, the subcellular simulation setup application provides a number of facilities for the visualization of models' geometries and the results of simulations.

To demonstrate the compatibility of the subcellular simulation setup application with the workflow for model development described above, we imported the SBML version of the use case model to the setup application and simulated it with the STEPS TetOpSplit solver. We have used a simple two-compartmental spine model with a tetrahedral compartment corresponding to the spine compartment of the use case model. There is also a PSD compartment on one of the
B

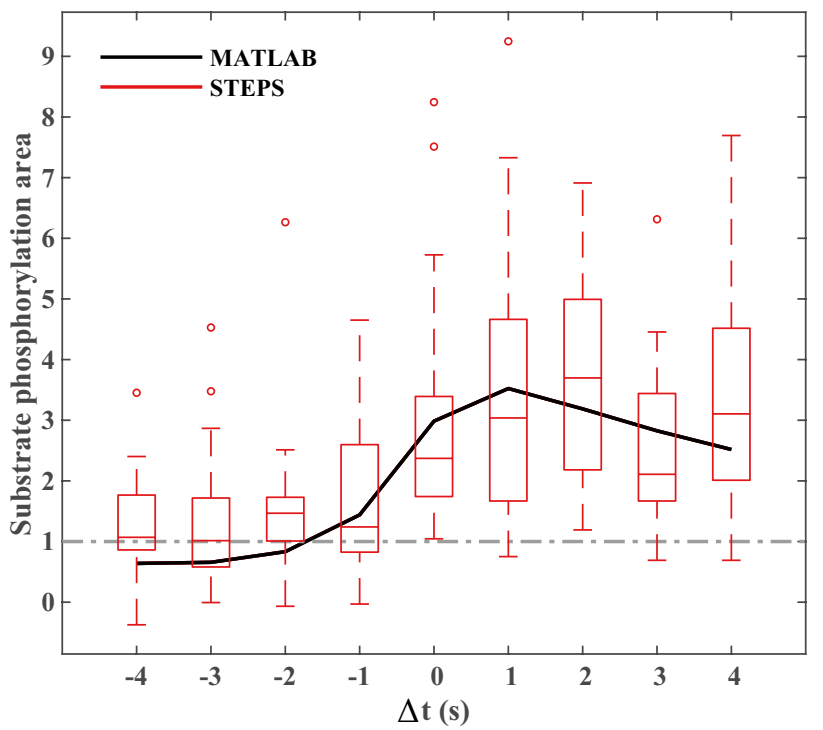

( 6000 molecules). B Normalized area under the curve of substrate phosphorylation with different calcium and dopamine input intervals simulated for the MATLAB ${ }^{\circledR}$ version of the updated model (with MATLAB ${ }^{\circledR}$ ode 15 s solver, black line) and averaged stochastic STEPS simulations $(n=30)$ in the application version of the model. MATLAB ${ }^{\circledR}$ statistical bar plots were added to the figure to characterize variability of synaptic plasticity between subsequent induction protocol applications to the same synaptic spine. Note that despite high variability of synaptic plasticity time courses averaged plasticity dynamics were in a good agreement with the ODE-based solution

faces. The results of the model simulations with a STEPS solver were qualitatively similar to the results obtained with the deterministic model simulated in MATLAB $®$. Examples of simulated time courses for molecule concentrations as shown in Fig. 3 in comparison with corresponding MATLAB ${ }^{\circledR}$ curves are shown in Fig. 6.

\section{Conversion to a MOD File and Simulations in NEURON}

As we suggested before, conversion between different modeling frameworks and formats facilitates collaboration. But conversion is made harder by the differences in the capabilities of different modeling packages. A model, such as the one above, could be useful for multiscale simulations investigating how neuronal network activity shapes synaptic plasticity. Many cellular level models are built and simulated in the NEURON environment which also supports simplified reaction-diffusion systems. It is useful to be able to integrate a subcellular level model into a cellular level model specified using NEURON. Models in NEURON are built by adding features with MOD files that are written in the NMODL programming language. A schematic illustration of our use case set up in NEURON is depicted in Fig. 7. Conversion from 
A

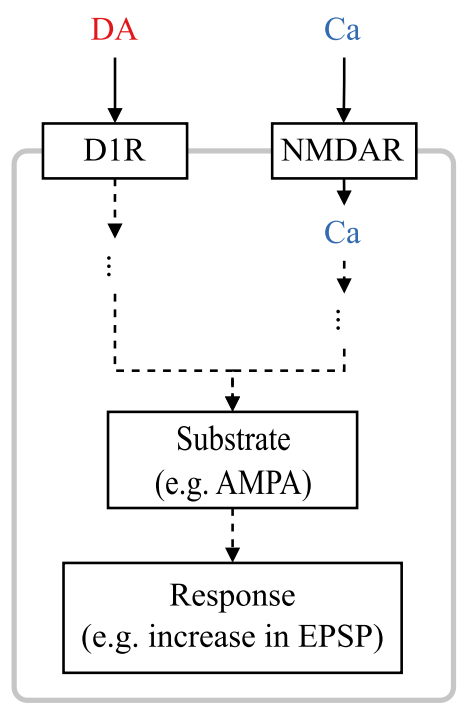

B
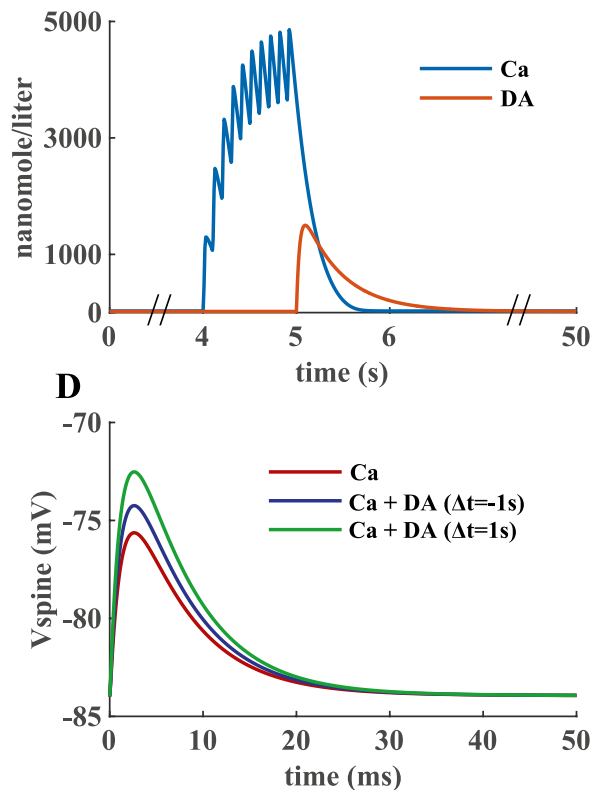

C
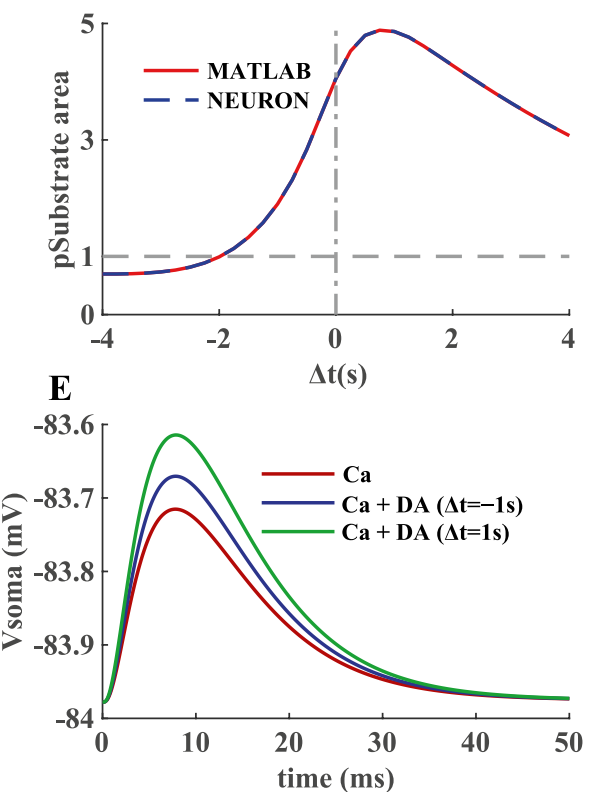

Fig. 7 Inserting the biochemical signal transduction cascade into an electrical model in NEURON. A A schematic of the effects of the two inputs of the model, dopamine and calcium, on a generic substrate, which in this case is taken to represent the fraction of phosphorylated AMPA receptors with higher conductance levels. B Examples of the two inputs, calcium and dopamine. The calcium signal at the synapse is a result of ten repeats of a synaptic stimulus paired with three somatic spikes evoked with a current clamp (Yagishita et al., 2014, Fig. 1). C The simulations in MATLAB and NEURON give the same results when using the same calcium input from the NEURON

SBML to MOD is already possible via NeuroML (instructions can be found in Lindroos et al., 2018); however, it is not an automated or user-friendly approach. We wrote an $\mathrm{R}$ script, which reads an SBtab model ${ }^{17}$ and writes a MOD file, as well as VFGEN and an SBML ${ }^{18}$ file. The script can optionally perform analysis of conservation laws and output a model where some of the differential equations for the state variables are substituted by algebraic equations arising from the conservation laws, thereby reducing the number of differential equations to be solved. Instructions on how to use the SBtab to VFGEN/MOD/SBML converter in $\mathrm{R}$ can be found in a separate GitHub repository ${ }^{19}$. The subcellular model in SBtab form is not aware of its coupling to a larger model of the cell and the user must edit the

\footnotetext{
$\overline{17}$ A file given either as a series of tab separated text files or one open document spreadsheet file. Some content of the SBtab model is mandatory, some optional.

18 libSBML must installed with $\mathrm{R}$ bindings: i.e. SBML output is optional.

19 Conversion function available in https://github.com/a-kramer/ SBtabVFGEN and instructions in https://github.com/a-kramer/ SBtabVFGEN/blob/master/README.md
}

simulation in MATLAB. This simulation in NEURON requires $4 \mathrm{~h}$ on 8 compute nodes on an Intel i7-4700MQ CPU @ $2.40 \mathrm{GHz}$. and less than one second in MATLAB ${ }^{\circledR}$ using an intel core i9-10980XE D, E Predicted EPSP (Excitatory PostSynaptic Potential) following a single synaptic input in the relevant spine and in the soma. The readout of the substrate phosphorylation level was done at $7 \mathrm{~s}$ after the start of the dopamine input. The relative timings of dopamine and calcium indicated in the figure legends are used, and the results are compared to the experimental setting without the dopamine input

resulting MOD file manually to use it within a larger scope and assign a role to this model component. This typically means assigning input to the model and using the output in some way. An example is given below with our use case. Another point to stress is that the script converts the time unit of the parameters to milliseconds, NEURON's default unit for time, but does not change the concentration units. Thus, when coupling a biochemical cascade to other quantities in the neuronal model, care must be taken to rescale the coupled variables so that they match the units in the rest of the neuronal model. We also illustrate that with one of the inputs in the use case.

We validate the biochemical cascade model and our conversion tools in NEURON by qualitatively reproducing the results obtained in MATLAB ${ }^{\circledR}$ SimBiology ${ }^{\circledR}$. Our goal is to show that the cascade model can be integrated into a single neuron model and bridge spatial and temporal scales of system behavior by linking the output of the cascade to changes in the synaptic properties and ultimately to the electrical behavior of the neuron model. Therefore, the biochemical model in the MOD format was incorporated into a single biophysically detailed and compartmentalized D1 MSN model from Lindroos et al. (2018). To integrate 
the MOD file into single cell models a few user-specific modifications have to be made to interface this model with the larger electrochemical system. First, the input is modified so that the calcium burst is represented by calcium influx from the cell's calcium channels and adjusted so that the overall calcium level would be similar to the input used in MATLAB $\AA$ simulations. The calcium in the neuron model is expressed in millimolar units and when coupling it to the biochemical cascade we rescale it to nanomolar units (the units used in the biochemical cascade model). The dopamine transient is represented by an assignment expression (available in the Expression table of the SBtab) that creates its double exponential form (this can be used in other languages as well). For an adequate comparison of the substrate phosphorylation curve (shown in Fig. 3D) obtained from the NEURON and MATLAB simulations it is necessary to provide the same input in the simulations. Hence, in the comparison in Fig. 7C we used the calcium input recorded from the NEURON simulation in the MATLAB simulation.

In the D1 MSNs this biochemical signaling cascade causes synaptic strengthening via several mechanisms, one of which is the phosphorylation of AMPA receptors. As mentioned above, the model instead includes a generic substrate whose level of phosphorylation is the output of the cascade. For the purpose of illustrating the workflow with a proof-of-concept example, we have here linked the fraction of phosphorylated substrate to the AMPA receptor conductance (the conductance is scaled by $1+f$ (where $f$ : fraction of the phosphorylated substrate), i.e. when there is very little substrate phosphorylation, very little change in the AMPA conductance is elicited, and vice versa. Modifying the model to include the reactions for AMPA receptor phosphorylation will be made in a future study.

\section{Discussion}

In order to address the growing need for interoperability in biochemical pathway modeling within the neuroscience field, we have developed a workflow that can be used to refine models in all phases of development, keeping in mind the fact that many of the users (including us) are scientists and not professional programmers. For the model and data storage we have chosen the SBtab format which can be easily read and modified by both modelers and experimentalists, and can be converted into other formats, e.g. SBML, MATLAB ${ }^{\circledR}$ SimBiology ${ }^{\circledR}$ or MOD. Our workflow is modularized into different steps allowing the use of each step depending on the need and ensuring interoperability with other tools, such as those described in a similar endeavor named FindSim (Viswan et al.,
2018). There are distinct advantages to the workflow, by enforcing a common standard for information exchange, it inherently makes the models more generalizable and reduces the likelihood that simulation results are artifacts of a particular simulator, and nonetheless, it gives users the flexibility to leverage the strengths of each different simulation environment and provides distinct stages of processing that would not be possible in any single simulator. The presented workflow aims to use software components that are free (apart from MATLAB $\AA$ ) and solve incremental sub-tasks within the workflow (with open standard intermediate files) to make the workflow easy to branch into scenarios we have not previously considered. It is also possible to circumvent MATLAB ${ }^{\circledR}$ entirely, if desired (e.g. conversion from SBtab to a MOD file that is then used by NEURON).

When deciding which software packages to use we find that an important aspect that must be considered is the cost and licensing. For some researchers, price may be a relevant concern, in other cases a researcher may have to undergo considerable overhead to make their institution/lab purchase a license and possibly operate a license server. Other than MATLAB $®$, we made the choice to disregard commercial products, keeping in line with the field's trend towards open source platforms. We will also expand our tools to support the use of models with more complex geometries, with several compartments (which is also an SBML feature), and tetrahedral meshes that can be used with STEPS in the subcellular simulation setup application. Such advanced geometries can in principle be defined within SBtab tables. When it comes to multiscale simulations, there is the possibility of using the Reaction-Diffusion module (RXD) in NEURON. Currently, however, it does not support the import of SBML as it lacks the concept of spatially extended models, but SBML support might be added to the future versions of RXD (McDougal et al., 2013).

Another interesting consideration is whether the user wants to define any model directly using rules (as in rulebased modeling). This would make the use of Atomizer unnecessary. The transformation from rules to classical reactions seems easier than the reverse, so even if a rulebased simulation is not necessary or too slow, a rule-based description may be shorter and more fundamental in terms of model translation.

When it comes to model analysis, we have here implemented a functionality for global sensitivity analysis. This is a thorough, but computationally demanding approach and the analysis usually needs to be run in parallel on a high performance computing environment. There are also faster but more approximate screening methods that could have been used (Saltelli, 2004). In the future we also intend to incorporate uncertainty quantification into the workflow (Eriksson et al., 2019). 
While the current workflow is standalone, there are potential alignments to other systems that could assist adoption by the community. For example, other systems such as Galaxy are more general in scope focusing on bioinformatics and are aimed at naive users (Afgan et al., 2016). The current workflow focuses on neuroscience and assumes experienced users. While the current version requires user installation of dependencies, in the future Dockerised versions could potentially help ease installation requirements. Similarly, the Common Workflow Language (Amstutz et al., 2016) is another recent development that could facilitate the exchange of workflow information.

In summary, we have here presented a workflow for biochemical pathway modeling and provided a concrete use case of reward dependent synaptic plasticity, with two additional examples in the supplementary material and the workflow repository. Multiscale models are crucial when trying to understand the brain using modeling and simulations, e.g. how network activity shapes synaptic plasticity or how neuromodulation might affect cellular excitability on sub second timescales. Structured approaches for bridging from detailed cellular level neuron models, to more simplified or abstract cellular-, network-, and even brain region models are developing (Amsalem et al., 2020; Carlu et al., 2020; Schmutz et al., 2020). In the currently illustrated workflow, we add to these efforts by bridging from the subcellular scale to the cellular level scale. We updated parts of the use case model to accomplish a model with only bimolecular reactions that are easier to represent in standards such as SBML. The idea is that users can look at our model as a concrete test case, rerun the workflow (or parts thereof) and then replace the current example model (Fujita et al., 2010, Hass et al., 2019) with their own models. In this particular use case, we specifically focused on creating scripts to achieve interoperability between human readable model specification standards and machine- readable standards, and we also wanted to facilitate how a subcellular signaling model could be implemented in different solvers with different strengths, in this case both SimBiology $\AA$ in MATLAB $®$, as well as STEPS and NEURON. NEURON is currently the most used simulation software for detailed cellular level neuron models, and STEPS can, as said, simulate signaling cascades in arbitrary dendritic morphologies both in a deterministic and stochastic manner. MATLAB ${ }^{\circledR}$ on the other hand has many functions, for example for parameter estimation and we included an implementation for global sensitivity analysis. Several other software is, however, used within the computational neuroscience community for cellular or subcellular model simulations (Akar et al., 2019; Oliveira et al., 2010; Ray \& Bhalla, 2008; Resasco et al., 2012). To successively make as many of those tools interoperable with standards for both model and data specification, various parameter estimation and model analysis methods, visualization software, etc., will further facilitate the creation of FAIR multiscale modeling pipelines in the future.

\section{Information Sharing Statement}

Source code of the Subcellular Workflow is available at https://github.com/jpgsantos/Subcellular_workflow/ tree/1.0 and licensed under GNU General Public License v3.0., documentation of the Subcellular Workflow is available at https://subcellular-workflow.readthedocs.io/en/1.0/. We stored the files relevant to each of the models in independent repositories, https://github.com/jpgsantos/Model_ Nair_2016/releases/tag/1.0 for the main model discussed in this paper, https://github.com/jpgsantos/Model_Viswan_ 2018/releases/tag/1.0 for the model discussed in the supplementary material, and https://github.com/jpgsantos/ Model_Fujita_2010/releases/tag/1.0 for the model mentioned in the supplementary material but mostly defined in the GitHub. The models are stored in the SBtab format (Lubitz et al., 2016). Model reduction, parameter estimation and global sensitivity analysis tools are written in MATLAB ${ }^{\circledR}$ (RRID:SCR_001622) and require the SimBiology® toolbox. Conversion script to VFGEN (Weckesser, 2008), MOD and SBML (RRID:SCR_007422) is written in R (RRID:SCR_001905). Conversion to SBML requires the use of libSBML (RRID:SCR_014134). Validations are run in COPASI (RRID:SCR_014260; Hoops et al., 2006), NEURON (RRID:SCR_005393; Hines \& Carnevale, 1997) and with the subcellular simulation setup application (RRID:SCR_018790; available at https://subcellular. humanbrainproject.eu/model/simulations) that uses a spatial solver provided by STEPS (RRID:SCR_008742; Hepburn et al., 2012) and network-free solver NFsim (available at http:// michaelsneddon.net/nfsim/). The medium spiny neuron model (Lindroos et al., 2018) used in NEURON simulations is available in ModelDB database (RRID:SCR_007271) with access code 237,653. The FindSim use case model is available in https://github.com/BhallaLab/FindSim (Viswan et al., 2018).

Supplementary Information The online version contains supplementary material available at https://doi.org/10.1007/s12021-021-09546-3.

Acknowledgements We thank Pavlo Getta for engineering support, Geir Halnes for sharing scripts for global sensitivity analysis with us, and Sahil Moza for helping with the FindSim use case. The simulations were partly performed on resources provided by the Swedish National Infrastructure for Computing (SNIC) at Lunarc.

Funding The study was supported by the Swedish research council (VR-M-2017-02806, VR-M-2020-01652), Swedish e-Science Research Centre (SeRC), EU/Horizon 2020 no. 785907 (HBP SGA2) and no. 945539 (HBP SGA3); EPFL Blue Brain Project Fund and the ETH Board Funding to the Blue Brain Project; scholarship PD/ BD/114180/2016 from FCT Fundação para a Ciência e Tecnologia.

Availability of Data and Material see Information Sharing Statement. 
Code Availability see Information Sharing Statement.

\section{Declarations}

Ethics Approval not applicable.

Consent to Participate not applicable.

Consent for Publication not applicable.

Conflicts of Interest/Competing Interests The authors declare that they have no conflict of interest.

Open Access This article is licensed under a Creative Commons Attribution 4.0 International License, which permits use, sharing, adaptation, distribution and reproduction in any medium or format, as long as you give appropriate credit to the original author(s) and the source, provide a link to the Creative Commons licence, and indicate if changes were made. The images or other third party material in this article are included in the article's Creative Commons licence, unless indicated otherwise in a credit line to the material. If material is not included in the article's Creative Commons licence and your intended use is not permitted by statutory regulation or exceeds the permitted use, you will need to obtain permission directly from the copyright holder. To view a copy of this licence, visit http://creativecommons.org/licenses/by/4.0/.

\section{References}

Afgan, E., Baker, D., van den Beek, M., Blankenberg, D., Bouvier, D., Čech, M., Chilton, J., Clements, D., Coraor, N., Eberhard, C., Grüning, B., Guerler, A., Hillman-Jackson, J., Von Kuster, G., Rasche, E., Soranzo, N., Turaga, N., Taylor, J., Nekrutenko, A., \& Goecks, J. (2016). The Galaxy platform for accessible, reproducible and collaborative biomedical analyses: 2016 update. Nucleic Acids Research., 44(W1), W3-W10.

Akar, N. A., Cumming, B., Karakasis, V., Küsters, A., Klijn, W., Peyser, A., \& Yates, S. (2019). Arbor - A morphologicallydetailed neural network simulation library for contemporary highperformance computing architectures. 27th Euromicro International Conference on Parallel, Distributed and Network-Based Processing (PDP), Pavia, Italy, pp. 274-282.

Amsalem, O., Eyal, G., Rogozinski, N., Gevaert, M., Kumbhar, P., Schürmann, F., \& Segev, I. (2020). An efficient analytical reduction of detailed nonlinear neuron models. Nature Communications, 11(1), 288.

Amstutz, P., Crusoe, M., Tijanić, N., Chapman, B., Chilton, J., Heuer, M., Kartashov, A., Leehr, D., Ménager, H., Nedeljkovich, M., Scales, M., Soiland-Reyes, S., \& Stojanovic, L. (2016): Common Workflow Language, v1.0. Specification, Common Workflow Language working group. https://w3id.org/cwl/v1.0/

Bhalla, U. S., \& Iyengar, R. (1999). Emergent properties of networks of biological signaling pathways. Science, 283(5400), 381-387.

Bhalla, U. S. (2004). Models of cell signaling pathways. Current Opinion in Genetics \& Development, 14, 375-381.

Bois, F. Y. (2009). GNU MCSim: Bayesian statistical inference for SBMLcoded systems biology models. Bioinformatics, 25, 1453-1454

Cannon, R. C., Gewaltig, M. -O., Gleeson, P., Bhalla, U. S., Cornelis, H., Hines, M. L., et al. (2007). Interoperability of neuroscience modeling software: Current status and future directions. Neuroinformatics, 5(2), 127-138.

Carlu, M., Chehab, O., Dalla Porta, L., Depannemaecker, D., Héricé, C., Jedynak, M., Köksal Ersöz, E., Muratore, P., Souihel, S., Capone, C., Zerlaut, Y., Destexhe, A., \& di Volo, M. (2020). A mean-field approach to the dynamics of networks of complex neurons, from nonlinear Integrate- and Fire to Hodgkin-Huxley models. Journal of Neurophysiology, 123(3), 1042-1051.

Chylek, L. A., Harris, L. A., Faeder, J. R., \& Hlavacek, W. S. (2015), Modeling for (physical) biologists: an introduction to rule-based approach. Physical Biology, 12(4), 045007.

Djurfeldt, M., Hjorth, J., \& Eppler, J. M., et al. (2010). Run-Time Interoperability Between Neuronal Network Simulators Based on the MUSIC Framework. Neuroinform, 8, 43-60. https://doi.org/10. 1007/s12021-010-9064-z.

Eriksson, O., Jauhiainen, A., Maad Sasane, S., Kramer, A., Nair, A. G., Sartorius, C., \& Hellgren Kotaleski, J. (2019). Uncertainty quantification, propagation and characterization by Bayesian analysis combined with global sensitivity analysis applied to dynamical intracellular pathway models. Bioinformatics, 35(2), 284-292.

Fujita, K. A., Toyoshima, Y., Uda, S., Ozaki, Y., Kubota, H., \& Kuroda, S. (2010). Decoupling of receptor and downstream signals in the Akt pathway by its low-pass filter characteristics. Science signaling, 3(132), ra56.

Gillespie, D. T. (1976). A General Method for Numerically Simulating the Stochastic Time Evolution of Coupled Chemical Reactions. Journal of Computational Physics, 22(4), 403-434.

Gillespie, D. T. (2001). Approximate accelerated stochastic simulation of chemically reacting systems. Journal of Chemical Physics, 115(4), 1716-1733.

Gleeson P, Crook S, Cannon RC, Hines ML, Billings GO, et al. (2010) NeuroML: A Language for Describing Data Driven Models of Neurons and Networks with a High Degree of Biological Detail. PLOS Computational Biology 6(6): e1000815. https://doi.org/10. 1371/journal.pcbi.1000815

Gleeson, P., Steuber, V., Silver, R. A., \& Crook, S. (2012). NeuroML. In: Le Novère N. (eds) Computational Systems Neurobiology. Springer, Dordrecht.

Haario, H., Laine, M., Mira, A., \& Saksman, E. (2006). DRAM: Efficient adaptive MCMC. Statistics and Computing, 16, 339-354.

Halnes, G., Ulfhielm, E., Eklöf Ljunggren, E., Hellgren Kotaleski, J., \& Rospars, J. P. (2009). Modelling and sensitivity analysis of the reactions involving receptor, G-protein and effector in vertebrate olfactory receptor neurons. Journal of Computational Neuroscience, 27(3), 471-491.

Harris, L. A., Hogg, J. S., Tapia, J. J., Sekar, J. A., Gupta, S., Korsunsky, I., Arora, A., Baruda, D., Sheehan, R. P., \& Faeder, J. R. (2016). BioNetGen 2.2: advances in rule-based modeling. Bioinformatics, 32(21), 3366-3368.

Hass, H., Loos, C., Raimndez-Alvarez, E., Timmer, J., Hasenauer, J., \& Kreutz, C. (2019). Benchmark problems for dynamic modeling of intracellular processes. Bioinformatics, 35, 3073-3082.

Hedley, W. J., Nelson, M. R., Bullivant, D. P., \& Nielsen, P. F. (2001). A short introduction to CellML. Philosophical Transactions of the Royal Society, Series A, 359, 1073-1089.

Hellgren Kotaleski, J., \& Blackwell, K. T. (2010). Modelling the molecular mechanisms of synaptic plasticity using systems biology approaches. Nature Reviews Neuroscience, 11, 239-251.

Hepburn, I., Chen, W., Wils, S., \& De Schutter, E. (2012). STEPS: Efficient simulation of stochastic reaction-diffusion models in realistic morphologies. BMC Systems Biology, 6, 36.

Hines, M. L., \& Carnevale, N. T. (1997). The NEURON simulation environment. Neural Computation, 9, 1179-1209.

Hoops, S., Sahle, S., Gauges, R., Lee, C., Pahle, J., Simus, N., Singhal, M., Xu, L., Mendes, P., \& Kummer, U. (2006). COPASI: A COmplex PAthway SImulator. Bioinformatics, 22, 3067-3074.

Hucka, M., Finney, A., Sauro, H. M., Bolouri, H., Doyle, J. C., Kitano, H., et al. (2003). The systems biology markup language (SBML): A medium for representation and exchange of biochemical network models. Bioinformatics, 19, 524-531. 
Klinger, E., Rickert, D., \& Hasenauer, J. (2018). pyABC: Distributed, likelihood-free inference. Bioinformatics, 34(20), 3591-3593.

Li, L., Stefan, M. I., \& Le Novere, N. (2012). Calcium input frequency, duration and amplitude differentially modulate the relative activation of calcineurin and CaMKII. PLoS One, 7, e43810.

Lindroos, R., Dorst, M. C., Du, K., Filipović, M., Keller, D., Ketzef, M., Kozlov, A. K., Kumar, A., Lindahl, M., Nair, A. G., PérézFernandez, J., Grillner, S., Silberberg, G., \& Hellgren Kotaleski, J. (2018). Basal ganglia neuromodulation over multiple temporal and structural scales-simulations of direct pathway MSNs investigate the fast onset of dopaminergic effects and predict the role of Kv4.2. Frontiers in Neural Circuits, 12, 3.

Lubitz, T., Hahn, J., Bergmann, F. T., Noor, E., Klipp, E., \& Liebermeister, W. (2016). SBtab: A flexible table format for data exchange in systems biology. Bioinformatics, 32(16), 2559-2561.

Maiwald, T., \& Timmer, J. (2008). Dynamical modeling and multiexperiment fitting with PottersWheel. Bioinformatics, 24(18), 2037-2043.

McDougal, R. A., Hines, M. L., \& Lytton, W. W. (2013). Reactiondiffusion in the NEURON simulator. Frontiers in Neuroinformatics, 7, 28 .

Nair, A. G., Bhalla, U. S., \& Kotaleski J. H. (2016). Role of DARPP-32 and ARPP-21 in the emergence of temporal constraints on striatal Calcium and Dopamine integration. PLoS Computational Biology, 12(9), e1005080.

Oliveira, R. F., Terrin, A., Di Benedetto, G., Cannon, R. C., Koh, W., Kim M., Zaccolo, M., \& Blackwell K. T. (2010). The role of type 4 phosphodiesterases in generating microdomains of cAMP: large scale stochastic simulations. PLoS One, 5(7), e11725.

Pepke, S., Kinzer-Ursem, T., Mihalas, S., \& Kennedy, M. B. (2010). A dynamic model of interactions of $\mathrm{Ca} 2+$, calmodulin, and catalytic subunits of $\mathrm{Ca} 2+/$ calmodulin-dependent protein kinase II. PLOS Computational Biology, 6(2), e1000675.

Raue, A., Kreutz, C., Maiwald, T., Bachman, J., Schilling, M., Klingmüller, U., \& Timer, J. (2009). Structural and practical identifiability analysis of partially observed dynamical models by exploiting the profile likelihood. Bioinformatics, 25(15), 1923-1929.

Ray, S., \& Bhalla, U. S. (2008). PyMOOSE: Interoperable scripting in Python and MOOSE. Frontiers in Neuroinformatics, 2, 6.

Resasco, D. C., Gao, F., Morgan, F., Novak, I. L., Schaff, J. C., \& Slepchenko, B. M. (2012). Virtual Cell: Computational tools for modeling in cell biology. Wiley Interdisciplinary Reviews: Systems Biology and Medicine, 4(2), 129-140.

Rodriguez, N., Pettit, J. -B., Dalle Pezze, P., Li, L., Henry, A., van Iersel, M. P., et al. (2016). The systems biology format converter. BMC Bioinformatics, 17, 154.

Saltelli, A. (2002). Making best use of model evaluations to compute sensitivity indices. Computer Physics Communications, 145, 280-297.

Saltelli, A. (2004). Sensitivity analysis in practice: A guide to assessing scientific models. Wiley.

Schmidt, H., \& Jirstrand, M. (2006). Systems Biology Toolbox for MATLAB: A computational platform for research in systems biology. Bioinformatics, 22(4), 514-515.
Schmutz, V., Gerstner, W., \& Schwalger, T. (2020). Mesoscopic population equations for spiking neural networks with synaptic shortterm plasticity. The Journal of Mathematical Neuroscience, 10(1), 5 .

Schälte, Y., Fröhlich, F., Stapor, P., Wang, D., Vanhoefer, J., Weindl, D., et al. (2020). ICB-DCM/pyPESTO: pyPESTO 0.2.0 (Version v0.2.0). Zenodo.

Sneddon, M. W., Faeder, J. R., \& Emonet, T. (2011). Efficient modeling, simulation and coarse-graining of biological complexity with NFsim. Nature Methods, 8(2), 177-183.

Sobol, I. M. (2001). Global sensitivity indices for nonlinear mathematical models and their Monte Carlo estimates. Mathematics and Computers in Simulation, 55(1-3), 271-280.

Tapia, J. J., \& Faeder, J. R. (2013). The Atomizer: extracting implicit molecular structure from reaction network models. In: Proceedings of the International Conference on Bioinformatics, Computational Biology and Biomedical Informatics (BCB'13), ACM, New York, NY, pp. 726-727.

Tennøe, S., Halnes, G., \& Einevoll, G. T. (2018). Uncertainpy: A python toolbox for uncertainty quantification and sensitivity analysis in computational neuroscience. Frontiers in Neuroinformatics, 14(12), 49.

Viswan, N. A., HarshaRani, G. V., Stefan, M. I., \& Bhalla, U. S. (2018). FindSim: A framework for integrating neuronal data and signaling models. Frontiers in Neuroinformatics, 12, 38.

Vlad, M. O., \& Ross, J. (1994). Thermodynamic approach to nonequilibrium chemical fluctuations. Journal of Chemical Physics, 100(10), 7295-7309.

Weckesser, W. (2008). VFGEN: A code generation tool. Journal of Numerical Analysis, Industrial and Applied Mathematics, 3(1-2), 151-165.

Wegscheider, R. (1901). Über simultane Gleichgewichte und die Beziehungen zwischen Thermodynamik und Reactionskinetik homogener Systeme. Monatshefte Für Chemie, 22, 849-906.

Welsh, C. M., Fullard, N., Proctor, C. J., Martinez-Guimera, A., Isfort, R. J., Bascom, C. C., Tasseff, R., Przyborski, S. A., \& Shanley, D. P. (2018). PyCoTools: A Python toolbox for COPASI. Bioinformatics, 34(21), 3702-3710.

Wilkinson, M. D., Dumontier, M., Aalbersberg, I. J., Appleton, G., Axton, M., Baak, A., ... \& Mons, B. (2016). The FAIR Guiding Principles for scientific data management and stewardship. Scientific data, 3(1), 1-9.

Yagishita, S., Hayashi-Takagi, A., Ellis-Davies, G. C. R., Urakubo, H., Ishii, S., \& Kasai, H. (2014). A critical time window for dopamine actions on the structural plasticity of dendritic spines. Science, 345(6204), 1616-1620. https://doi.org/10.1126/science.1255514

$\mathrm{Zi}, \mathrm{Z}$. (2011). Sensitivity analysis approaches applied to systems biology models. IET Systems Biology, 5(6), 336-346.

Publisher's Note Springer Nature remains neutral with regard to jurisdictional claims in published maps and institutional affiliations. 